\title{
CTRP4 binds to the interleukin- 6 Receptor and ameliorates autoimmune encephalomyelitis by suppressing Th17 cell differentiation
}

\section{Lulu Cao}

Center for Human Disease Genomics

\section{Wei Chen}

Center for Human Disease Genomics

\section{Daxiang $\mathrm{Na}$}

Center for Human Disease Genomics

\section{He Huang}

Center for Human Disease Genomics

Qi Li

Institute of Chinese Materia Medica

\section{Xiaoxin Zhu}

Institute of Chinese Materia Medica, China Academy of Chinese Medical Sciences

Lu Wang ( $\nabla$ wanglu@bjmu.edu.cn )

Center for Human Disease Genomics

\section{Article}

Keywords: CTRP4,autoimmune,Th17, IL-6R,

Posted Date: March 26th, 2021

DOl: https://doi.org/10.21203/rs.3.rs-265573/v1

License: (9) (i) This work is licensed under a Creative Commons Attribution 4.0 International License. Read Full License 


\section{Abstract}

CTRP4, a secreted protein, plays an important role in protecting against sepsis and energy metabolism; however, its physiological functions in autoimmune disease remain unknown. In this study, we demonstrate that Th17 cell-associated experimental autoimmune encephalomyelitis was greatly exacerbated in $\mathrm{Ctrp}^{-/-}$mice because of increased Th17 cell infiltration. In vitro, Ctrp4 deficiency enhanced the ability of naïve $\mathrm{CD} 4^{+} \mathrm{T}$ cells to differentiate into Th17 cells. Mechanistically, CTRP4 interfered with the binding of IL-6 to its receptor IL-6R by directly binding to IL-6R and suppressed the activation of STAT3-related pathways in response to IL-6. Furthermore, treatment of induced EAE mice with recombinant CTRP4 protein ameliorated the symptom. In conclusion, our results indicate that CTRP4, as an endogenous regulator of the IL- 6 receptor signaling pathway, may be a potential therapeutic intervention for multiple sclerosis.

\section{Introduction}

Multiple sclerosis is an inflammatory disorder of the central nervous system (CNS) identified by chronic demyelination and axonal damage. Upon activation in the peripheral lymphoid organs, the autoimmune $T$ cells enter the CNS through the blood brain barrier and are reactivated, resulting in the infiltrated leukocytes and disseminated inflammation, demyelination and severe disease symptoms ${ }^{1,2,3}$. One of the most frequent animal models of multiple sclerosis is experimental autoimmune encephalomyelitis $(E A E)^{3}$. Studies using EAE model suggest that the initiation of EAE is associated with peripheral priming of myelin-specific autoimmune Th1 and Th17 cells ${ }^{2}$. Furthermore, dysregulated Th17 cell activity has been shown to induce the pathogenesis of multiple sclerosis and correlate with disease severity and relapse frequency ${ }^{4}$.Th17 cells display considerable plasticity and are categorized as pathogenic or nonpathogenic depending on the cytokine milieu. TGF $\beta$ and IL- 6 drive classical Th17 cell differentiation ${ }^{5,6}$, while Th17 cells with pathogenic functions are poised to develop strong tissue-destructive properties upon on IL-23 and IL-1 $\beta$ expression, which induce the expression of the effector cytokines GM-CSF and IFNy. These cell populations impair the blood brain barrier and promote neuronal damage ${ }^{7,8}$.

Interleukin-6 (IL-6) is a key cytokine to initiate the transcriptional program of Th17 cells, which is indispensable for the EAE disease process. IL- 6 signaling is activated by the formation of a complex between IL-6 and different forms of IL-6R. Several modes of gp130 activation are initiated and mainly recruit STAT3, which is followed by downstream signaling activation. Classic signaling is triggered by IL- 6 binding to the membrane form of IL-6R. Soluble IL-6R is cleaved from membrane-bound IL-6R by the proteases ADAM10 and ADAM17 or is produced upon translation of an alternatively spliced IL6R mRNA 10. IL-6 binds to soluble IL-6R and is required for cells that do not express IL-6R on the surface, a process termed trans-signaling. A third mode termed as trans-presentation has been identified: dendritic-cellspecific IL-6 and membrane IL-6R are presented to gp130-expressing T cells and induce pathogenic Th17 cells $^{11,12}$. Interfering with the IL-6-STAT3 signaling axis appears to have effects on the disease progression of EAE in different ways ${ }^{13}$. 
C1q/TNF-related protein 4(CTRP4), featuring two highly conserved complement C1q domains connected by a short linker, is a classical secreted protein of 329 amino acids ${ }^{14}$. CTRP4 played a critical role in a DSS-induced mouse colitis-associated colorectal cancer model by mediating the inflammatory response ${ }^{15}$. Loss of protective functions in a CTRP4-deficient sepsis model through promoting of TLR4 internalization, activation of downstream pathways and inflammatory cytokine release in macrophages ${ }^{16}$. There is also evidence suggesting that CTRP4 generated by neurons plays a role in energy metabolism by modulating food intake and body weight ${ }^{17,18}$. These results suggested that CTRP4 is an anti-inflammatory cytokine, but the precise mechanism underlying its role in the autoimmune disease is unknown.

This study was prompted by our observation that immunized $\mathrm{Ctrp}^{-/-}$mice immunized to induce EAE exhibited severe symptoms. We investigated this molecular mechanism starting from the finding that CTRP4 suppressed IL-6-mediated STAT3 activation by interacting with IL-6R, interfering with the binding of IL-6 to its receptor, IL-6R. In addition, we elucidated the treatment efficacy of CTRP4 in EAE. This study has important implications in the understanding of CTRP4 function and the development of MS therapy.

\section{Methods}

\section{Experimental Mice}

The generation of $\mathrm{Ctrp}^{-/-}$mice has been previously described ${ }^{16}$. To produce I/6ra-knock out mice, we crossed $/ 16 \mathrm{ra}^{\mathrm{fl} / \mathrm{fl}}$ mice with EllaCre, in which IL-6R is depleted in all tissues. B6.SJL mice were purchased from the Jackson Laboratory. All mouse strains had a C57BL/6J background, and six- to twelve-week-old mice were used for most of experiments. Littermates with the appropriate genotypes were used as ageand sex-matched controls. All mice were bred and maintained under SPF conditions in an animals facility.

All animal experiments were conducted according to the guidelines of the Institutional Animal Care.

\section{EAE model establishment:}

The $M O_{35-55}$ peptide with the amino acid sequence MEVGWYRSPFSRVVHLYRNGK was synthesized by Synpeptide. We immunized 8-to10-week-old female mice subcutaneously with $200 \mu \mathrm{g}$ of $\mathrm{MOG}_{35-55}$ emulsified with incomplete Freund's adjuvant (Cat\#F5506, Sigma) containing $5 \mathrm{mg} / \mathrm{mL}$ heat-killed Mycobacterium tuberculosis (Cat\#231141, BD Bioscience), followed by an tail vein injection of $200 \mathrm{ng}$ of pertussis toxin (Cat\#179B, List Biological Laboratories)on day 0. An intraperitoneal injection of $200 \mathrm{ng}$ of pertussis toxin was administered on day 2. The mice were examined daily for clinical signs of disease and evaluated as previously described ${ }^{19}$.

\section{Induction of EAE by passive transfer of pathogenic $\mathrm{CD4}^{+} \mathrm{T}$ cells}

B6.SJL donor mice (CD45. $1^{+}$) were immunized by subcutaneous injection of $200 \mu \mathrm{g}$ MOG peptide emulsified with CFA including $5 \mathrm{mg} / \mathrm{mL}$ heat-killed Mycobacterium tuberculosis distributed over four 
spots on the flank. Ten days later, draining lymph node cells were isolated and re-stimulated with 50 $\mu \mathrm{g} / \mathrm{mL}$ of MOG and Th17 cell-polarizating factors $(10 \mathrm{ng} / \mathrm{mL}$ rhIL-23, $10 \mathrm{ng} / \mathrm{ml} \mathrm{IL-} 1 \mathrm{a}$ and anti-IL4 and antiIFN-y) to generate Th17 cells. After 4 days in culture, the resting cells were sorted with anti-CD 4 microbeads (Miltenyi, Tokyo), and calculate the same number of Th17 cells ( $1 \times 10^{6} /$ mouse) was calculated and then give intravenously injected into irradiated C57/BL6J recipient mice (4 Gy). The mice were injected with $200 \mathrm{ng}$ pertussis toxin in PBS on day 0 and 2 days after transfer. The mice were observed daily for signs of clinical disease following EAE induction and scored using a scale previously described.

\section{Th17 cell differentiation}

Naïve $\mathrm{CD} 4^{+} \mathrm{T}\left(\mathrm{CD} 4^{+} \mathrm{CD} 44^{\mathrm{lo}} \mathrm{CD} 62 \mathrm{~L}^{\text {hi }} \mathrm{CD} 25^{-}\right.$) cells from the spleen and lymph nodes were purified by flow cytometry, and the purity of the isolated T cell population was nearly $98 \%$. Recombinant mouse IL- 6 , mouse TGF $\beta$, and mouse IL-6/IL-6R protein chimaeras were purchased from R\&D Systems. Recombinant mouse IL-23, and mouse IL-1 a were purchased from PeproTech. Naïve T cells were stimulated with platebound anti-CD3 $(2 \mu \mathrm{g} / \mathrm{mL})$ and anti-CD28 $(2 \mu \mathrm{g} / \mathrm{mL})$ antibodies in the presence of anti-IL4 and anti-IFN- $\gamma$ to generate Th0 cells; IL-6 (30 ng/ml), TGF- $\beta 1(2 \mathrm{ng} / \mathrm{ml})$, anti-IL4 and anti-IFN- $\gamma$ antibodies were added to induce Th17 cell differentiation. Pathogenic Th17 cells were generated in the presence of IL- $6(30 \mathrm{ng} / \mathrm{ml})$,

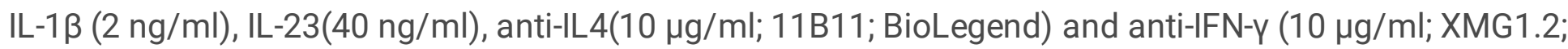
BioLegend). At the end of the culture period, we re-stimulated $C D 4^{+} T$ cells with $P M A(50 \mathrm{ng} / \mathrm{mL})$, ionomycin $(1 \mu \mathrm{g} / \mathrm{mL})$ in the presence of monensin $(10 \mu \mathrm{g} / \mathrm{mL})$ for $4 \mathrm{~h}$ for intracellular staining. For mRNA expression, cells were harvested $4 \mathrm{~h}$ after PMA and ionomycin stimulation.

Flow cytometry and related reagents: Single-cell suspensions were isolated from LNs, the spleen and the CNS by mechanical isolation. The brain and spinal cord were removed and homogenized and then incubated with $0.5 \mathrm{mg} / \mathrm{mL}$ collagenase type I and $1000 \mathrm{U} / \mathrm{mL}$ DNasel for $45 \mathrm{~min}$. Mononuclear cells were enriched by gradient centrifugation at $670 \mathrm{~g}$ for $30 \mathrm{~min}$ on a $37 / 70 \%$ Percoll gradient without interruption. The $37 / 70 \%$ interphase was collected with a transfer pipette following centrifugation, and washed with a threefold volume of $1 \% \mathrm{RPMI}$, and then centrifuged for $10 \mathrm{~min}$ at $350 \mathrm{~g}$ to collect leukocytes. For intracellular cytokine staining, cells were incubated with anti-CD16/CD32 before being stained. The following antibodies were used for the flow cytometry: anti-CD4 (GK1.5), anti-CD8 (53 - 6.7), anti-CD25 (PC61.5), anti-CD62L (MEL-14), anti-CD44 (IM7), anti-Foxp3 (FJK-16S), anti-Ly-6G/Gr-1 (1A8-Ly6g), antiGM-CSF (MP1-22E9), anti-IL-17A (eBio17B7), and anti-IFN-y (XMG1.2) were from eBioscience and anti-IL2 (JES6-5H4), anti-pSTAT3 (Y705, 557814), pSTAT1 (Y701, 502069) were from BD Bioscience. After staining of surface markers, the cells were fixed and permeabilized according to the manuscript's instructions. Phosphoflow cytometry analysis was performed using BD Phosflow buffers (554655 and 558050). Stained cells were analyzed using a FACSCanto flow cytometer, and the data were analysed with FlowJo software.

\section{Quantitative RT-PCR}


Total RNA samples were extracted with Trizol reagent and reverse transcribed to cDNA according to the manufacturer's insructions.

Quantitative RT-PCR was performed using SYBR Green (Transgene) with a Roche LightCycler480 system. All the primers used for real-time PCR are listed in supplemental table 1. The conditions for real-time PCR were 40 cycles at $94^{\circ} \mathrm{C}$ for $15 \mathrm{~s}$ followed by 40 cycles at $60^{\circ} \mathrm{C}$ for $60 \mathrm{~s}$. The target gene expression levels were normalized to Gapdh mRNA levels.

\section{Western blot analysis}

Cell lysates were prepared in RIPA buffer containing protease inhibitor cocktail and phosphatase inhibitor cocktail, and were centrifuged at $3500 \mathrm{rpm}$ for $10 \mathrm{~min}$ at $4^{\circ} \mathrm{C}$. Proteins were subjected to PAGE with $10 \%$ gels and transferred to nitrocellulose membranes and probed with antibodies against phospho-STAT3 (CST, Cat. no. \#9145, 1:1000 dilution), STAT3 (CST, Cat.no.\#4904,1:1000), phospho-JAK2 (CST, Cat.no.\#3771, 1:1000 dilution), phospho-ERK (CST, Cat.no.\#4370, 1:1000 dilution), ERK(CST, Cat.no.\#4695, 1:1000 dilution), phosphor-Akt (CST, Cat.no.\#4060, 1:1000 dilution), Akt (CST, Cat.no.\#4691, 1:1000 dilution), IL-6R (Santa Cruz, Cat.no.sc373708, 1:1000 dilution), IL-6 (CST, Cat.no.\#12153, 1:1000 dilution), anti-FLAG (sigma-Aldrich; Cat.no.F3165, 1:1000 dilution) and anti-c-Myc (Sigma-Aldrich, Cat.no.C3956, 1:1000 dilution). The membranes were then incubated with appropriate secondary antibody and developed with Amersham ECL (GE Healthcare, Uppsala, Sweden).

\section{Immunoprecipitation}

Immunoprecipitation was performed as we previously described ${ }^{20}$. Briefly, various cells were lysed in an lysis buffer (20 mM Tris-HCl, pH 7.5; 150 mM NaCl; 0.5 mM EDTA; 1.5 mM MgCl 2 ; 0.1\%NP-40; 10\% glycerol and protease inhibitor cocktail) at $4^{\circ} \mathrm{C}$ for $30 \mathrm{~min}$. The lysates were centrifuged for $10 \mathrm{~min}$ at $13,000 \times \mathrm{g}$. Protein concentrations were determined, and then equal amounts of cell lysates were immunoprecipitated with anti-IL6R, or anti-CTRP4 antibodies bound to protein G Sepharose (GE Healthcare), or anti-FLAG M2 Affinity Gel (Cat\#A2220,Sigma). After extensive washing five times with lysis buffer, the immunoprecipitates were subjected to SDS-PAGE gel.

\section{Radio-ligand binding assay}

CTRP4 protein was purified from CTRP4-CHO cells as described ${ }^{16}$. CTRP4 was labelled with ${ }^{125} \mathrm{I}$ in $0.01 \mathrm{~N}$ PB buffer with chloramine $\mathrm{T}$ at $4^{\circ} \mathrm{C}$ for $30 \mathrm{~s}$, followed by elution with $0.01 \mathrm{~N}$ PB buffer in a SEPHADEXG25 column. For saturation experiments, equivalent quantities of Jurkat cell membrane extract were incubated with a 2 -fold serial dilution of [ ${ }^{125}$ l]-CTRP4 in binding buffer. Nonspecific binding was measured in the presence of a 500-fold excess of CTRP4 at each concentration of [ $\left.{ }^{125}\right]$ ]-CTRP4. For competition-binding assays, equivalent quantities of cell membrane extract and [125]]-CTRP4 were incubated with different concentrations of unlabelled CTRP4, OSM or IL-6. The reaction was incubated for $24 \mathrm{hr}$ at $4^{\circ} \mathrm{C}$ with shaking, and $25 \% \mathrm{PEG}$ was added, followed by counting on a $y$ counter.

\section{ELASA}


Human IL-6R protein (R\&D) was precoated onto plates and maintained overnight at $4^{\circ} \mathrm{C}$ and then incubated with human IL- 6 or CTRP4 protein in duplicate. The plates were washed twice with PBS $/ 0.05 \%$ Tween 20. Each well in the plates was blocked with $200 \mu$ of $1 \%$ BSA in PBS for $2 \mathrm{~h}$. The plates were incubated with anti-IL-6R primary antibody. After washing the plates, detection was performed by streptavidin-HRP and tetramethylbenzidine (TMB). The plate was monitored for 10-20 min for color change and read at OD405. Supernatants from cell cultures were collected after activation under different conditions and secreted cytokines in the supernatants were measured by CBA assay (Biolegend).

\section{Histological assay of spinal cord sections}

Diseased animals were perfused at the peak of disease with cold PBS followed by $4 \%$ paraformaldehyde for fixation. The spinal cords were dissected and fixed with 4\% PFA, dehydrated and embedded in paraffin. $5 \mu \mathrm{m}$-thick sections were stained with haematoxylin \& eosin or Luxol fast blue. For immunofluorescence staining with anti-CD 4 and anti-IL-17, sections were incubated at $55^{\circ} \mathrm{C}$ for $30 \mathrm{~min}$ for antigen retrieval. The sections were pretreated with a $0.3 \%$ solution of $\mathrm{H}_{2} \mathrm{O}_{2}$ to block endogenous peroxidase activity. The sections were then incubated with $10 \%$ goat serum in PBS-T at room temperature for $30 \mathrm{~min}$. The sections were then incubated with primary antibodies diluted in blocking buffer for $24 \mathrm{~h}$ at $4^{\circ} \mathrm{C}$. The primary antibodies were anti-IL-17A (Invitrogen, 14-7179-80, 1:100 dilution) and anti-CD4 (Invitrogen, 14-9766-82, 1:100 dilution). The sections were labelled with biotin-tagged anti-lgG (1:200 in PBS-T; Vector Laboratories), followed by incubation with avidin-biotin complex (Vector Laboratories) and biotinylated tyramide. Antibody binding was detected with Alexa Fluor 555-labelled streptavidin against IL-17 at room temperature for $1 \mathrm{~h}$. After staining with DAPI to visualize cell nuclei, the slides were mounted and analysed by fluorescence microscopy.

\section{Lentiviral infection}

IL-6R and gp130 cDNA was cloned into a lentivirus vector TG006. For lentivirus production, HEK293T cells were transfected with $10 \mu \mathrm{g}$ of TG006-gp130 transfer vector in $1.5 \mathrm{~mL}$ of opti-MEM and $5 \mu \mathrm{g}$ of psPAX2 and $5 \mu \mathrm{g}$ of VSVG packing vector. Lentivirus particles were harvested $72 \mathrm{~h}$ after transfection, filtered and added to $\mathrm{Ba} / \mathrm{F} 3$ cells in the presence of polybrene $(8 \mu \mathrm{g} / \mathrm{ml})$. After $24 \mathrm{~h}$, the medium containing the viral particles was replaced by viral particle-free DMEM medium. The cells were cultured and maintained in DMEM containing $2 \mu \mathrm{g} / \mathrm{ml}$ puromycin.

\section{Statistical analysis}

Experimental results were analysed for significance using one-way ANOVA or Student's t-test assuming unequal variance, except for the EAE clinical data, which were analysed by Mann-Whitney $U$ test on each day. Statistical analyses were performed using GraphPad Prism, version 6.0. P-values $<0.05$ were considered statistically significant.

\section{Results}

Increased numbers of Th17 cells in the periphery of $\mathrm{Ctrp4}^{-/-}$mice 
In our previous study, we already observed preferential expression of Ctrp4 in T cells, which inspired us to further analyse the function of Ctrp4 in T cell development and differentiation. To this end, we first

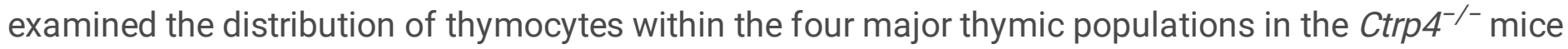
and WT controls. We found that eight-week-old CTRP4-deficient mice had undetectable changes in the percentage of $\mathrm{CD} 4^{+} \mathrm{T}$ cells and had normal thymocyte development, suggesting a minimal role of CTRP4 in $T$ cell development(Fig. 1A-B). Considering that the size of the peripheral $\mathrm{T}$ cell pool is stably maintained under homeostatic conditions and that the most of peripheral $\mathrm{T}$ cells are in a naïve state with $\mathrm{CD} 44^{\mathrm{lo}} \mathrm{CD} 62 \mathrm{~L}^{\mathrm{hi}}$, a proportion of memory-like $\mathrm{T}$ cells, characterized by CD44 ${ }^{\text {hi }} \mathrm{CD} 62 \mathrm{~L}^{\text {lo }}$ markers, increase with age because of homeostatic proliferation induced by self-peptide/MHC ligands. Notably, a marked increase in memory-like $\mathrm{CD} 4^{+} \mathrm{T}$ cells in $\mathrm{Ctrp} 4^{-/-}$mouse and a reduction in naïve $\mathrm{CD} 4^{+} \mathrm{T}$ cells were observed, which commonly tend to indicate autoimmune inflammation (Fig. 1C). However, the flow cytometry analysis showed no differences in the proportion of memory-like $\mathrm{CD} 8^{+} \mathrm{T}$ in the spleen(Fig. 1D).Then, we examined the effector T cell subsets in the periphery of WT and Ctrp4 ${ }^{-/}$- mice. Among the $\mathrm{CD} 44^{\text {hi }}$ memory $\mathrm{T}$ cell population in the spleen, the IL-17A-producing Th17 cell subset was profoundly increased in the Ctrp $4^{-/-}$mice (Fig. 1E), while the numbers of Treg, Th1 and Th2 cells remained unchanged when compared with those in the WT mice (Fig. 1E, supplemental Fig. 1A-B). Changes in different $\mathrm{CD} 4^{+} \mathrm{T}$ cell subsets were further measured by Th cell signature genes in $\mathrm{CD} 4^{+} \mathrm{T}$ cells. When $\mathrm{Ctrp}^{{ }^{-/-}}$T cells were compared with WT cells, we found that the mRNA expression of Th17 cell lineage-specific genes (I/17a, I/17f, Rorc and $\mathrm{CxCl3}$ ) was upregulated significantly (Fig. 1F), whereas Tbx21, Gata3 and Foxp3 gene expression was unaltered (supplemental Fig. 1C). In summary, these data suggest that CTRP4 may be involved in T cells differentiation toward the Th17 cell lineage and suggest the possibility that CTRP4 plays a role in autoimmunity.

\section{CTRP4 deficiency in mice exacerbates EAE symptom}

T cell responses to self-antigens contribute to the development of many autoimmune and inflammatory diseases. To gain further insight into the pathophysiological roles of CTRP4 in the Th17 cell-mediated autoimmune disease, we generated the EAE models with CTRP4-sufficient and -deficient mice by immunizing them with myelin oligodendrocyte glycoprotein $\left(\mathrm{MOG}_{35}-55\right)$ to mimic human $\mathrm{MS}$. Compared with the wild-type mice, disease progression in the $\mathrm{Ctrp4}^{-/-}$mice showed advanced onset and developed more severe clinical symptoms and more body weight loss, suggesting a role for CTRP4 in reducing EAE induction (Fig. 2A). Haematoxylin and eosin or Luxol fast blue staining revealed that more immune cell infiltration and increased demyelination and axon degeneration in the spinal cord (Fig. 2B). To investigate the population of lymphocytes infiltrating the CNS, we isolated mononuclear cells from the CNS. The immunohistological analysis and flow cytometry showed more $\mathrm{CD} 4^{+} \mathrm{T}$ cells in neuronal tissue in the $\mathrm{Ctrp}^{-/-}$mice 18 days after immunization (Fig. 2C, supplemental Fig. 2A). The phenotype of Ctrp4knockout mice was characterized by an increased frequency and absolute number of CNS-infiltrating $\mathrm{CD} 45^{+} \mathrm{F} 4 / 80^{+}$macrophages during the peak phase of EAE (Fig. 2D). Additionally, the number of IL17A and IL $17 \mathrm{~A}^{+}$IFNY ${ }^{+}$double-producing $\mathrm{CD} 4^{+} \mathrm{T}$ cells, which reflects the disease severity, was increased in 
Ctrp4-deficient mice as compared with the number in the spinal cords from CTRP4-expression counterparts (Fig. 2E-F, supplemental Fig. 2B). Furthermore, the infiltration of Th1 and Treg cells was not different between WT and CTRP4-deficient mice (Fig. 2E). To investigate whether the augment of CNSinfiltrating $\mathrm{CD}^{+}$cells in the $\mathrm{Ctrp} 4^{-/-}$mice was due to increased peripheral Th17 cells or not, we further analysed the proportions of $\mathrm{CD} 4^{+} \mathrm{T}$ cells and Th17 cells in the draining lymph nodes and spleen. Increased frequencies of Th17 cells corresponded with an increased number of CNS-infiltrating Th17 cells at the peak of the EAE symptoms, suggesting that CTRP4 may influence the number of Th17 cells not only in the CNS but also in the peripheral lymphoid tissues (supplemental Fig. 2D-G).

To determine the role of Ctrp4 in regulating the recall response of $\mathrm{MOG}_{35}-55^{-}$-specific $T$ cells, we stimulated T cells isolated from draining LNs at the early effector phase of EAE induction and cultured them ex vivo. Upon re-stimulation with MOG peptides, we found that $\mathrm{MOG}_{35}-55^{\text {-stimulated }} \mathrm{T}$ cells exhibited substantially increased proliferation in the Ctrp4-deficient mice (Fig. 2G). Consistently, the production of IL-17 and IFN- $\gamma$ was significantly increased in the $C t r p 4^{-/-}$mice, which correlated with the clinical score (Fig. $2 \mathrm{H}$ ). To further elucidate the subsets of $\mathrm{MOG}_{35}-55^{-}$peptide-specific $\mathrm{CD} 4^{+} \mathrm{T}$ cells,

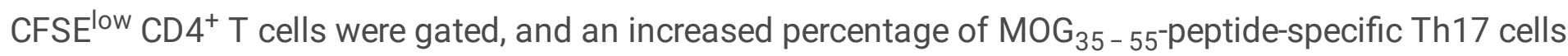
was observed in sample obtained from CTRP4-deficient mice (Fig. 2I). We thus speculated that the loss of CTRP4 promotes EAE disease progression primarily by regulating Th17 cells.

\section{CTRP4 deficiency facilitates IL-6-driven Th17 cell differentiation}

We further examined whether the increased accumulation of Th17 cells in the CNS in Ctrp4 ${ }^{-/-}$mice was attributed to the different abilities of Th17 cells to migrate, expand, survive, or infiltrate in the absence of CTRP4. We first sorted and cultured naïve CD $4^{+} \mathrm{T}$ cells obtained from $\mathrm{Ctrp4}^{-{ }^{-}-}$and WT mice and cultured them under classical Th17 cell-polarizing condition and analysed their cytokine profiles. The loss of Ctrp4 resulted in a significant difference in the percentage of IL17A-producing cells and increased the production of IL-17A in the cultures (Fig. 3A). Consistent with this finding, CD4 ${ }^{+} \mathrm{T}$ cells lacking of Ctrp4 exhibited increased Rorc mRNA expression under Th17 cell-differentiating conditions, while $C t r p 4^{-/-} \mathrm{T}$ cells remained unchanged under Th0 cell-differentiating conditions (Fig. 3B). Moreover, IL-23 is critical for the differentiation of pathogenic Th17 cells. Exposure of naïve $\mathrm{CD} 4^{+} \mathrm{T}$ cells to the cytokine combination of IL-1 $\beta+$ IL- $6+$ IL-23 results in the acquisition of a pathogenic Th17 cell phenotype. Thus, we next examined whether CTRP4 deficiency affected the differentiation of pathogenic Th17 cells. More IL-17producing $\mathrm{CD}^{+}$cells from $\mathrm{Ctrp} 4^{-/-}$mice were observed under pathogenic conditions than were observed among the cells obtained from WT mice, and IL-17A was much more abundant in the $\mathrm{Ctrp}^{-/-}$cultures than in the control cultures (Fig. 3A). Th17-associated genes, such as Rorc, I/17a and $/ 117 f$, were significantly upregulated in the $\mathrm{Ctrp}^{-/-} \mathrm{CD} 4^{+} \mathrm{T}$ cells, implying that elevated IL-17 secretion can be partially attributed to the increased expression of their mRNAs. In addition, IL23R expression on T cells is essential for Th17 cell stability and pathogenicity. The mRNA expression of II23rand Ifng was also increased in $\mathrm{CD}^{+} \mathrm{T}$ cells from the $\mathrm{Ctrp}^{-/-}$mice (Fig. $3 \mathrm{C}$ ). 
Next, we examined whether Ctrp4 deficiency affects T cell proliferative capacity. We performed flow cytometry analysis of CFSE dilution in $\mathrm{CD}^{+} \mathrm{T}$ cells stimulated with TCR activation or Th17 cell-polarizing

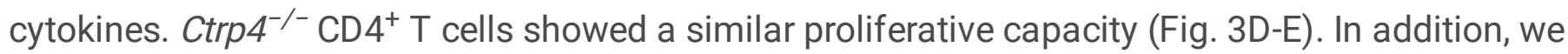
evaluated the apoptotic capacity of CTRP4-deficient CD4 ${ }^{+} \mathrm{T}$ cells under the same conditions, and similarly, no significant differences was detected (Fig. 3F-G). With regard to Th17 cell migration, Th17 cells highly express the chemokine receptors CCR6 and CCR2, which allow their recruitment to the CNS 21 , 22. Our data demonstrate that there was no difference in the proportion of cells that expressed CCR2 and CCR6 after Ctrp4 was depleted, suggesting that Ctrp4 did not alter the ability of Th17 cells to traffic to sites of inflammation (supplemental Fig. 3A). Consistent with this finding, the CNS of EAE-induced Ctrp4deficient expressed levels of various chemokines that mediate immune-cell recruitment that were similar to levels expressed by WT- immunized mice (supplemental Fig. 3B). Further, the absence of Ctrp4 in Th17 cells did not lead to changes in the expression of the key activation markers CD25, CD44, CD69 or CD103 (supplemental Fig. $3 \mathrm{C}$ ). Collectively, these findings further support the possibility that $\mathrm{Ctrp} 4$ directs $\mathrm{CD} 4^{+} \mathrm{T}$ cell fate choice toward Th17 cell differentiation without affecting Th17 cell proliferation, apoptosis, migration or activation.

\section{CTRP4 inhibits IL-6 activation by directly binding with IL-6R}

Many studies have reported that IL- 6 signaling is required for the differentiation of Th17 cells, and we hypothesized that CTRP4 probably interacts with IL-6R to suppress IL-6-induced Th17 cell differentiation. We used computational molecular docking experiments performed using Discovery Studio software to analyse the interaction modes on the basis of X-ray crystal structure of IL-6R obtained from the PDB database. The docking results supported our hypothesis (supplemental Fig. 4A). To test this hypothesis, we performed confocal immunofluorescence microscopy. The results revealed CTRP4 colocalization with IL-6R in both the cytoplasm and membrane of Jurkat cells (Fig. 4A). The direct interaction was further confirmed by CTRP4 and IL-6R co-immunoprecipitating from primary $\mathrm{CD} 4^{+} \mathrm{T}$ cells, and reverse immunoprecipitation experiments showed the same results (Fig. 4B). To confirm interactional binding, recombinant CTRP4 protein was labelled with an ${ }^{125} \mathrm{I}$ tracer. Saturation-binding assays demonstrated the direct binding between CTRP4 and IL-6R with a $\mathrm{K}_{\mathrm{D}}$ of $3.941 \mathrm{nM}$ (Fig. 4C,F). We then evaluated the ability of unlabelled CTRP4, IL-6 and OSM of gp130 family to replace ${ }^{125}$ I-labelled CTRP4 in the competitionbinding assays. Indeed, unlabelled CTRP4 and IL- 6 strongly competed with radio-iodinated CTRP4 for binding with IL-6R with an $\mathrm{IC}_{50}$ value of $77.25 \mathrm{nM}$ and $5.233 \mathrm{nM}$, respectively, while OSM did not exert a competitive effect (Fig. 4D). To determine the region of IL-6R necessary for binding CTRP4, HEK293T cells were transfected with Myc-tagged CTRP4 and different FLAG-tagged domains of IL-6R. Because the D3 domain of IL-6R covers the majority of the IL-6 interface area ${ }^{23,24}$, the D3 domain was found to be critical for the IL-6R association with CTRP4, as expected (Fig. 4E).

While these results clearly suggest that CTRP4 interacts with IL-6R, we sought to investigate whether CTRP4 disturbed the formation of the IL-6-to-IL-6R complex for further activation. Thus, HEK293T cells transfected with pmCherry-tagged IL- 6 and EGFP-tagged IL-6R, and the significant colocalization was observed in the cytoplasm and membrane. However, the association was decreased in the presence of 
CTRP4 (Fig. 4H). This results was consistent with the ELASA results. The addition of recombinant CTRP4 protein impaired the interaction of IL-6 and IL-6R in an in vitro binding assay (Fig. 4G). Similarly, coimmunoprecipitation demonstrated that CTRP4 interfered with the binding of IL-6 to IL-6R (Fig. 4I).Collectively, these results demonstrated that CTRP4 may function to suppress IL-6/IL-6R signaling pathway activation during Th17 cell differentiation.

\section{CTRP4 prevents IL-6-mediated STAT3 phosphorylation by interacting with IL-6R}

IL6 induces Th17 cell differentiation through the activation of the STAT3 pathway ${ }^{25,26}$, especially the phosphorylation of STAT3 at tyrosine $705^{27,28}$. To delineate the molecular mechanism underlying the observed inhibitory effect of CTRP4 on Th17 cell differentiation, we assessed the signaling activity of JAK/STAT pathways. First, we determined that the expression of IL-6R and gp130 was comparable between WT and Ctrp4-deficient CD4 ${ }^{+}$T cells upon TCR stimulation (supplemental Fig. 4A). Furthermore, IL6 stimulation led to increased levels of pSTAT3 in WT cells, and we observed significantly higher levels of pSTAT3 at tyrosine 705 in Ctrp $^{-/-}$CD $4^{+}$T cells stimulated for 5 and 15 min with physiological concentrations of IL6 (Fig. 5A). Consistent with the results, enhanced STAT3 phosphorylation in the $\mathrm{Ctrp4}^{-/-} \mathrm{T}$ cells was observed by flow cytometry (Fig. 5B). Among the other signaling molecules tested, pJAK2, pERK and pAkt had higher expression than CD4 ${ }^{+}$T cells from WT mice, suggesting that the IL6STAT3 signaling pathway is constitutively hyper-activated after the loss of CTRP4 in CD $4^{+} \mathrm{T}_{\text {cells }}$ (Fig. 5A). To address the molecular mechanisms by which CTRP4 inhibits the development of EAE in vivo, we assessed the expression of the IL6 receptor in the context of the EAE model. Flow cytometry analysis revealed that the expression of IL6R and gp130 in the CD4 ${ }^{+} \mathrm{T}$ cells from CTRP4-deficient mice was similar to that in the $\mathrm{CD}^{+} \mathrm{T}$ cells from WT mice (supplemental Fig. 4B-D). Of note, the deficiency of CTRP4 in $\mathrm{CD}^{+} \mathrm{T}$ cells resulted in a significant increase in Y705-phosphorylated STAT3 in the spinal cord tissue of EAE mice compared to the level in the WT mice, while the abundance of phosphorylated STAT1 in the $\mathrm{CD} 4^{+} \mathrm{T}$ cells was not altered (Fig. $5 \mathrm{C}$ ).

Previous studies have shown that the IL6 signaling cascade is initiated by the binding of IL6 to membrane-bound IL6R and gp130, which is called a classical signal ${ }^{11}$. First, we used Ba/F3 cells, which are an IL-3-dependent mouse pro-B-cell line that lacks both endogenous IL-6R and gp130 expression 29, 30, to establish cells with gp130 and IL-6R through stable retroviral expression, and then we analysed their proliferation rate in response to IL-6 signaling (supplemental Fig. 5A). IL-6 alone induced the proliferation of Ba/F3-gp130-IL-6R cells, while rhCTRP4 substantially reduced IL-6-mediated proliferation, suggesting that CTRP4 affects classical IL-6 signaling (Fig. 5D). Furthermore, we investigated the effect of rhCTRP4 on IL- 6 trans-signaling. After establishing stable expression of gp130 in Ba/F3 cells, the cells proliferated in response to hyper-IL-6 but not in response to IL-6 (supplemental Fig. 5B-C). Exogenous CTRP4 suppressed hyper-IL-6-mediated Ba/F3-gp130 growth (Fig. 5E). This results was validated with MEFs obtained from IL-6R-knockout mice, which do not express membrane-bound IL-6R; therefore, IL-6 stimulation relies on trans-signaling (Fig. 5F). Recombinant CTRP4 protein treatment reduced basal pSTAT3 levels, and reversed IL-6- or hyper-IL-6- induced STAT3 activation (Fig. 5G). Together, these results 
suggested that CTRP4 influences proliferation by competing with IL-6 for membrane-bound or soluble IL$6 \mathrm{R}$.

\section{6. rhCTRP4 is effective for the treatment of EAE}

As CTRP4 is a secreted protein, we first verified whether the addition of rhCTRP4 impairs Th17 cell differentiation. The frequency of the IL17-producing cell population in culture was decreased in the presence of rhCTRP4 to the culture in vitro (Fig. $6 \mathrm{~A})$. The $\mathrm{CD} 4^{+} \mathrm{T}$ cells treated with rhCTRP4 under Th17 cell-differentiating conditions significantly reduced the Rorc and I/17a transcript levels (Fig. 6B), as also illustrated by a markedly decreased IL17A generation (Fig. 6C). To determine whether the degree of the suppressive effect correlated with the dose of rhCTRP4, we treated $\mathrm{CD} 4^{+} \mathrm{T}$ cells with various concentrations of rhCTRP4. The results indicated that higher doses of rhCTRP4 inhibited Th17 cell differentiation to a lesser extent, suggesting that rhCTRP4 impaired the differentiation of naïve $\mathrm{CD} 4^{+} \mathrm{T}$ cells towards Th17 cells in a dose-dependent manner (Fig. 6D-E). Consistent with the fact that CTRP4 had an impact on the IL-6 downstream pathway, pretreatment with rhCTRP4 abrogated STAT3 activation in naïve CD $4^{+}$T cells in response to IL6 (Fig. 6F). Additionally, the IL-6-triggered STAT3 phosphorylation increase was abolished as early as $5 \mathrm{~min}$ after exposure to rhCTRP4 and sustained the inhibitory effect through 60 min (Fig. 6G). Similarly, the enhanced phosphorylation of JAK2 and STAT3 by IL- 6 can be attenuated in a dose-dependent manner to normal levels by adding rhCTRP4 (Fig. 6H).Taken together, these results further confirmed that the IL6/STAT3 signaling is negatively regulated by CTRP4.

Next, we wondered whether rhCTRP4 is effective in alleviating established EAE disease in Ctrp4 ${ }^{-/-}$mice. We immunized mice with the MOG peptide, followed by daily intraperitoneal injection of rhCTRP4 on the day of immunization. As illustrated in Fig. 7A, treatment with rhCTRP4 showed significant efficacy and resulted in reduced severity of EAE compared with that observed in control mice. The histological analysis of the spinal cord revealed that administration of rhCTRP4 was accompanied by decreased inflammation and demyelination in the affected spinal cord (Fig. 7B). The mice had a marked reduction in the number of $\mathrm{CD} 4^{+}$cells and Th17 cells infiltrating the CNS upon therapeutic rhCTRP4 administration (Fig. 7C-D). The immunohistological analysis of pSTAT3 in the spinal cord revealed a reduced number of pSTAT3-positive cells in rhCTRP4-treated mice (Fig. 7E). Accordingly, treatment with rhCTRP4 suppressed the phosphorylation of STAT3 and JAK2 in CD4 ${ }^{+}$T cells at the peak of the disease (Fig. 7F). We also isolated $T$ cells from primed mice and detected their reactivity towards antigen. When challenged with the MOG peptide, the T cells derived from the lymph nodes of the EAE mice treated with rhCTRP4 displayed a markedly dampened proliferative response (Fig. 7G).IL-17A and IFNY generation was reduced in the MOGprimed T cells from rhCTRP4-injected mice (Fig. 7H). In summary, therapeutic delivery of rhCTRP4 ameliorated the clinical severity of EAE associated with reduced encephalitogenic effector T cells responses.

\section{CTRP4 prevents EAE disease in a STAT3-dependent manner}

We have shown that Th17 cells accumulate in $\mathrm{Ctrp4}^{-/-}$mice in a manner dependent on the activation of amplified IL-6. We wondered whether the suppressive effect of CTRP4 on STAT3 activation contributes to 
the protection of the host resistant to EAE. To target STAT3 signaling, we used S3I-201, which is a smallmolecule inhibitor that interacts with the STAT3-SH2 domain to suppress STAT3 homodimer formation and STAT3 binding to DNA ${ }^{31}$. After immunizing Ctrp4-Knockout mice with MOG peptide, the mice were treated either with DMSO or with S3I-201. The results showed that S3I-201 administration resulted in the mitigation of the autoimmune disease compared with disease level in the control mice (Fig. 8A), as reflected by a significant reduction in immune cell infiltration in the spinal cord (Fig. 8B). Similar to the inhibitory effects of CTRP4 in vitro, inhibition of STAT3 has a significant impact on ameliorating disease caused by the depletion of CTRP4 in vivo.

\section{MOG-reactive T cells that expand in the presence of rhCTRP4 ameliorated EAE}

We next determined whether CTRP4 can influence the pathogenicity of the autoantigen-specific CD $4^{+} T$ cell response in vivo. For this purpose, we immunized B6.SJL mice with $\mathrm{MOG}_{35-55}$ emulsified in CFA. Ten days later, we isolated lymphoid cells from the spleen and draining lymph nodes before re-stimulating the cells with MOG peptide, IL-23 and either BSA or rhCTRP4. Compared to the CD4 ${ }^{+} T$ cells stimulated with $B S A$, the $C D 4^{+} T$ cells treated with rhCTRP4 showed significantly reduced the frequency of MOG-specific Th17 cells. In agreement with the flow cytometry data, IL-17A release was diminished in the culture supernatants of the CD4 ${ }^{+}$T cells treated with IL-23 and rhCTRP4 (Fig. 8C).

To further determine whether rhCTRP4 incubation affects the pathogenicity of MOG-reactive CD4 ${ }^{+} T$ cells, expanded MOG-reactive $\mathrm{CD} 4^{+} \mathrm{T}$ cells were adoptively transferred into irradiated $\mathrm{C} 57 \mathrm{BL} / 6 \mathrm{~J}$ mice. The majority of the recipient mice injected T cells stimulated with rhCTRP4 exhibited significantly milder symptoms than the mice injected with BSA-treated T cells (Fig. 8D). We observed that the numbers of host $\mathrm{CD} 4^{+} \mathrm{T}$ cells and $\mathrm{CD} 4^{+} \mathrm{CD} 45.1^{+}$donor $\mathrm{T}$ cells were both comparable in the CNS in the two groups(Fig. 8E, F).Consistent with this finding, the frequency and absolute number of IL-17-producing cells among the CD45. $1^{+}$donor T cell population stimulated with IL-23 plus rhCTRP4 were similar to those of the donor T cells stimulated with IL-23 plus BSA. Notably, given that the comparable number of CD45. $1^{+}$CD $4^{+}$IL-17A ${ }^{+}$donor T cells, we speculate that Th17 cell pathogenic phenotype was impaired. Accordingly, the IL-17 producer were able to co-express GM-CSF and IFNY, an ability that was linked to the encephalitogenic potential of the Th17 cells and that was reduced in the recipients of the T cells treated with exogenous rhCTRP4 (Fig. 8E, G). Collectively, these results showed that pretreatment with rhCTRP4 during the ex vivo expansion of MOG-reactive $\mathrm{CD} 4^{+} \mathrm{T}$ cells rendered the $\mathrm{T}$ cells more encephalitogenic and more proficient in inducing autoimmune CNS inflammation.

\section{Discussion}

Previous studies showed that, CTRP4, as a secreted protein, conferred protection against endotoxic shock and diminished the susceptibility to the development of acute colitis and colitis-associated colorectal cancer ${ }^{17}$. Here, we demonstrated the immunomodulatory properties of CTRP4 in the IL-17-related autoimmune disease EAE, on which, to our knowledge, no functional reports exist to date. In the present study, the protective function of CTRP4 is highlighted by the finding that a lack of CTRP4 leads to much 
more severe clinical signs of EAE symptoms and histopathological severity, as well as increased frequency of IL-17A ${ }^{+}$and IFNY ${ }^{+}$IL- $17 A^{+}$T cells in the CNS. In the absence of CTRP4, similarly increased levels of accumulated Th17 cells were simultaneously observed in the CNS and periphery after EAE induction. This phenotype may be explained by the possibility that Ctrp4 acts directly on naïve CD4 ${ }^{+} \mathrm{T}$ cell to drive their differentiation towards Th17 cell lineage but does not influence the proliferation or apoptosis of Th17 cells. In line with this notion, a lack of Ctrp4 did not alter the expression of CCR6 or CCR2 in CD4 ${ }^{+} \mathrm{T}$ cells, which are known to be critical for the migration of Th17 cells into the CNS when local inflammation occurs. MOG-reactive T cells that were expanded in the presence of rhCTRP4 triggered less profound symptoms when the passive EAE induction approach was used. Hence, our findings confirmed the role of CTRP4 functions as an anti-inflammatory factor ${ }^{14,15,16,32}$.

Providing a molecular explanation for why CTRP4 modulates Th17 cell differentiation, we identified a previously unknown interaction between CTRP4 and IL-6R. Activation of STAT3 by IL- 6 requires IL- 6 binding to IL-6R to form the heterodimeric IL-6-IL-6R complex that interacts with gp 130 .In this study, we found that deficiency of Ctrp4 did not affect the expression levels of IL-6R or gp130 on the cell surface, suggesting that this is not the mechanism by which CTRP4 affects IL- 6 activity. The coimmunoprecipitation and radio-ligand binding assay results indicated that CTRP4 binds with IL-6R. In addition, we found that CTRP4 antagonized the association of IL- 6 with IL-6R by interacting with IL-6R and impaired the IL-6-mediated activation of STAT3, which is an essential regulator of the lineage commitment to Th17 cells ${ }^{33}$. This notion is also supported by additional pieces of evidence, such as the ELASA results showing that CTRP4 abrogated the interaction of IL-6 and IL-6R. Although the binding affinity between IL-6R and CTRP4 was relatively low compared with the affinity of IL-6 for IL-6R, a higher CTRP4 concentration in the serum compared with that of IL- 6 affords more opportunity for CTRP4 to bind. Although our results support the conclusion that the greatly exacerbated EAE symptoms of $\mathrm{Ctrp4}^{-/-}$ mice were due to alterations in the differentiation of $\mathrm{CD} 4^{+} \mathrm{T}$ cells, the suppressive effect of CTRP4 cannot extended to other IL-6R-expressing cells, including immune cells and nonimmune cells. Further study is needed to gain a full understanding of the role of CTRP4 in multiple cell types and the universal role of the CTRP4 and IL-6R interaction in inflammatory disease.

The biological effects of IL- 6 are highly complex, as they can be mediated via multiple pathways. Classical IL-6 signaling primarily involves gp130 to activate downstream pathways upon membranebound IL-6R stimulation. Soluble IL-6R binding IL-6 induces signaling and takes place in cells that do not express IL-6R through trans-signaling ${ }^{34}$. Our data reveal that CTRP4 disturbs not only the classical pathway but also trans-signaling. Previous studies have shown that IL-6 bound to mIL-6R expressed on dendritic cells is presented to gp130-expressing T cells through trans-presentation ${ }^{35}$. Because rhCTRP4 can suppress Th17 cell differentiation in the absence of dendritic cells, we ruled out the possibility of IL6 trans-presentation in the process. It is possible that CTRP4 is involved in the plasma-cell differentiation of B cells or T follicular helper cells given the critical role of IL-6, and further studies will be needed to investigated these pathways. 
STAT3 is specifically required for the response to IL- 6 and plays a crucial function in CD $4+$ T cells differentiation ${ }^{36}$. At the molecular level, STAT3 stimulates the expression of the Th17 cell lineagespecifying RORY and RORa, which are required for Th17 development ${ }^{37}$. Our findings suggest that CTRP4 suppressed the activation of STAT3 in $\mathrm{CD}^{+} \mathrm{T}$ cells in vitro and in vivo. The fact that severe symptoms in $\mathrm{Ctrp}^{-/-}$mice can be attenuated in the presence of a STAT3 inhibitors was further confirmed, suggesting that CTRP4 plays a role in EAE in a STAT3-dependent manner. Thus, CTRP4 limited naïve CD $4^{+} \mathrm{T}$ cell he differentiation toward Th17 cell lineage.

Because suppression of IL-6R is an attractive strategy for the treatment of autoimmune diseases involving autoreactive immune cells, we wanted to know whether rhCTRP4 injection is effective for the treatment of EAE. In consistent with the results from Ctrp4-knockout mice, when rhCTRP4 was administered by intraperitoneal injection, a beneficial treatment effect was observed. In summary, CTRP4 is a promising candidate of the medicine for the treatment of multiple sclerosis.

\section{Declarations}

\section{Acknowledgements}

We thank Professor Dalong Ma and Professor Xiaoyu Hu for their thoughtful comments and review of this manuscript. We thank Professor Wenwen Zeng for kindly providing $\| 6 \mathrm{rafl} / \mathrm{fl}$ mice. We thank Professor Yingxian Li for kindly providing Ella-cre mice. This work was supported by grants from the National Natural Science Foundation of China. (No. 91542106 and NO.91129707).

\section{Author contributions}

C.L designed and performed experiments, analysed results and wrote the manuscript. C.W. and N.D.X conducted the experiments and analysed data.L.Q , Z.X.X and W.Lsupervised the study and writing of the manuscript.

\section{Competing interest}

All authors declare that they have no conflict of interest and have submitted the ICMJE Form for Disclosure of Potential Conflicts of Interest.

\section{References}

1. Goverman J. Autoimmune T cell responses in the central nervous system. Nature reviews Immunology 9, 393-407 (2009).

2. Korn T, Kallies A. T cell responses in the central nervous system. Nature reviews Immunology 17, 179-194 (2017).

3. Baecher-Allan C, Kaskow BJ, Weiner HL. Multiple Sclerosis: Mechanisms and Immunotherapy. Neuron 97, 742-768 (2018). 
4. Moser T, et al. The role of TH17 cells in multiple sclerosis: Therapeutic implications. Autoimmunity Reviews 19, 102647 (2020).

5. Bettelli E, et al. Reciprocal developmental pathways for the generation of pathogenic effector TH17 and regulatory T cells. Nature 441, 235-238 (2006).

6. Mangan PR, et al. Transforming growth factor-beta induces development of the $T(H) 17$ lineage. Nature 441, 231-234 (2006).

7. Huppert J, et al. Cellular mechanisms of IL-17-induced blood-brain barrier disruption. Faseb j 24, 1023-1034 (2010).

8. Kebir $\mathrm{H}$, et al. Human $\mathrm{TH} 17$ lymphocytes promote blood-brain barrier disruption and central nervous system inflammation. Nature medicine 13, 1173-1175 (2007).

9. Riethmueller S, et al. Proteolytic Origin of the Soluble Human IL-6R In Vivo and a Decisive Role of NGlycosylation. PLoS biology 15, e2000080 (2017).

10. Lust JA, et al. Isolation of an mRNA encoding a soluble form of the human interleukin-6 receptor. Cytokine 4, 96-100 (1992).

11. Quintana FJ. Old dog, new tricks: IL-6 cluster signaling promotes pathogenic TH17 cell differentiation. Nature Immunology 18, 8-10 (2017).

12. Heink $S$, et al. Trans-presentation of IL-6 by dendritic cells is required for the priming of pathogenic TH17 cells. Nature Immunology 18, 74-85 (2017).

13. Ciofani M, et al. A validated regulatory network for Th17 cell specification. Cell 151, 289-303 (2012).

14. Wang L. CTRP4: a new member of the adipocytokine family. Cellular \& molecular immunology 14 , 868-870 (2017).

15. Byerly MS, et al. C1q/TNF-related protein 4 (CTRP4) is a unique secreted protein with two tandem $\mathrm{C} 1 \mathrm{q}$ domains that functions in the hypothalamus to modulate food intake and body weight. $\mathrm{J} \mathrm{Biol}$ Chem 289, 4055-4069 (2014).

16. Cao L, et al. CTRP4 acts as an anti-inflammatory factor in macrophages and protects against endotoxic shock. European journal of immunology, (2020).

17. Luo Y, et al. Expression of the novel adipokine C1qTNF-related protein 4 (CTRP4) suppresses colitis and colitis-associated colorectal cancer in mice. Cellular \& molecular immunology 13, 688-699 (2016).

18. Li Y, et al. C1q/TNF-related Protein 4 Induces Signal Transducer and Activator of Transcription 3 Pathway and Modulates Food Intake. Neuroscience 429, 1-9 (2020).

19. Ogura $\mathrm{H}$, et al. Interleukin-17 promotes autoimmunity by triggering a positive-feedback loop via interleukin-6 induction. Immunity 29, 628-636 (2008).

20. Feng $\mathrm{H}$, et al. EGFR phosphorylation of DCBLD2 recruits TRAF6 and stimulates AKT-promoted tumorigenesis. J Clin Invest 124, 3741-3756 (2014).

21. Reboldi A, et al. C-C chemokine receptor 6 -regulated entry of TH-17 cells into the CNS through the choroid plexus is required for the initiation of EAE. Nature Immunology 10, 514-523 (2009). 
22. Kara EE, et al. CCR2 defines in vivo development and homing of IL-23-driven GM-CSF-producing Th17 cells. Nature Communications 6, 8644 (2015).

23. Boulanger MJ, Chow DC, Brevnova EE, Garcia KC. Hexameric structure and assembly of the interleukin-6/IL-6 alpha-receptor/gp130 complex. Science 300, 2101-2104 (2003).

24. Lacroix $M$, et al. Novel Insights into Interleukin 6 (IL-6) Cis- and Trans-signaling Pathways by Differentially Manipulating the Assembly of the IL-6 Signaling Complex. J Biol Chem 290, 2694326953 (2015).

25. Bromberg J, Wang TC. Inflammation and cancer: IL-6 and STAT3 complete the link. Cancer Cel/ 15, 79-80 (2009).

26. Yu H, et al. Revisiting STAT3 signalling in cancer: new and unexpected biological functions. Nature reviews Cancer 14, 736-746 (2014).

27. Renner ED, et al. Novel signal transducer and activator of transcription 3 (STAT3) mutations, reduced $\mathrm{T}(\mathrm{H}) 17$ cell numbers, and variably defective STAT3 phosphorylation in hyper-IgE syndrome. The Journal of allergy and clinical immunology 122, 181-187 (2008).

28. Guanizo AC, Fernando CD, Garama DJ, Gough DJ. STAT3: a multifaceted oncoprotein. Growth factors (Chur, Switzerland) 36, 1-14 (2018).

29. Palacios R, Steinmetz M. II-3-dependent mouse clones that express B-220 surface antigen, contain Ig genes in germ-line configuration, and generate B lymphocytes in vivo. Cell 41, 727-734 (1985).

30. Baran P, et al. Minimal interleukin 6 (IL-6) receptor stalk composition for IL-6 receptor shedding and IL-6 classic signaling. J Biol Chem 288, 14756-14768 (2013).

31. Siddiquee K, et al. Selective chemical probe inhibitor of Stat3, identified through structure-based virtual screening, induces antitumor activity. Proc Natl Acad Sci U S A 104, 7391-7396 (2007).

32. Aletaha D, Smolen JS. Diagnosis and Management of Rheumatoid Arthritis: A Review. Jama 320, 1360-1372 (2018).

33. Tanaka S, et al. Sox 5 and c-Maf cooperatively induce Th17 cell differentiation via RORyt induction as downstream targets of Stat3. The Journal of experimental medicine 211, 1857-1874 (2014).

34. Garbers C, Rose-John S. Dissecting Interleukin-6 Classic- and Trans-Signaling in Inflammation and Cancer. Methods Mol Biol 1725, 127-140 (2018).

35. Heink S, et al. Trans-presentation of IL-6 by dendritic cells is required for the priming of pathogenic $\mathrm{T}(\mathrm{H}) 17$ cells. Nat Immunol 18, 74-85 (2017).

36. Durant L, et al. Diverse targets of the transcription factor STAT3 contribute to T cell pathogenicity and homeostasis. Immunity 32, 605-615 (2010).

37. Ivanov, II, et al. The orphan nuclear receptor RORgammat directs the differentiation program of proinflammatory IL-17 + T helper cells. Cell 126, 1121-1133 (2006).

\section{Figures}



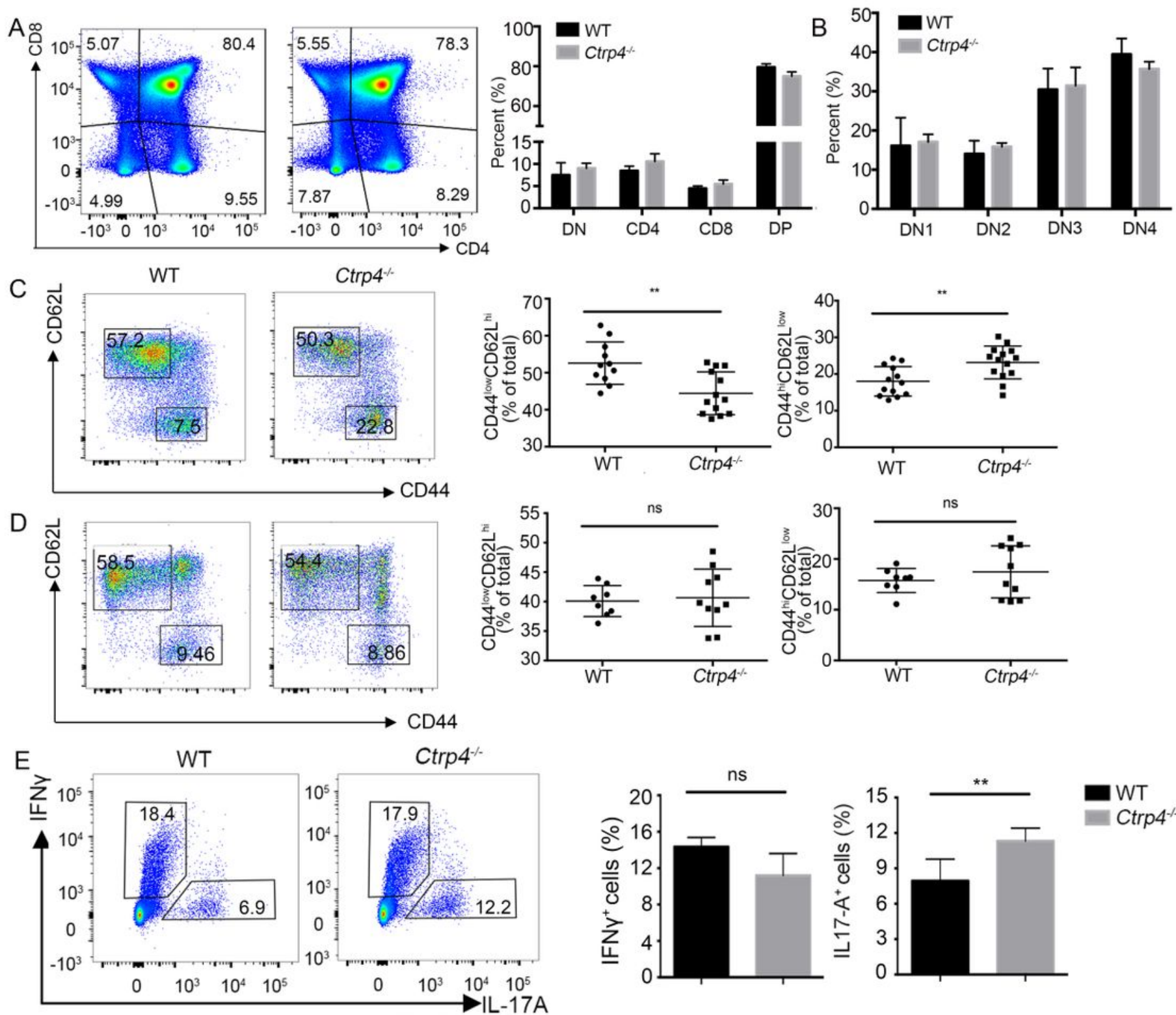

F
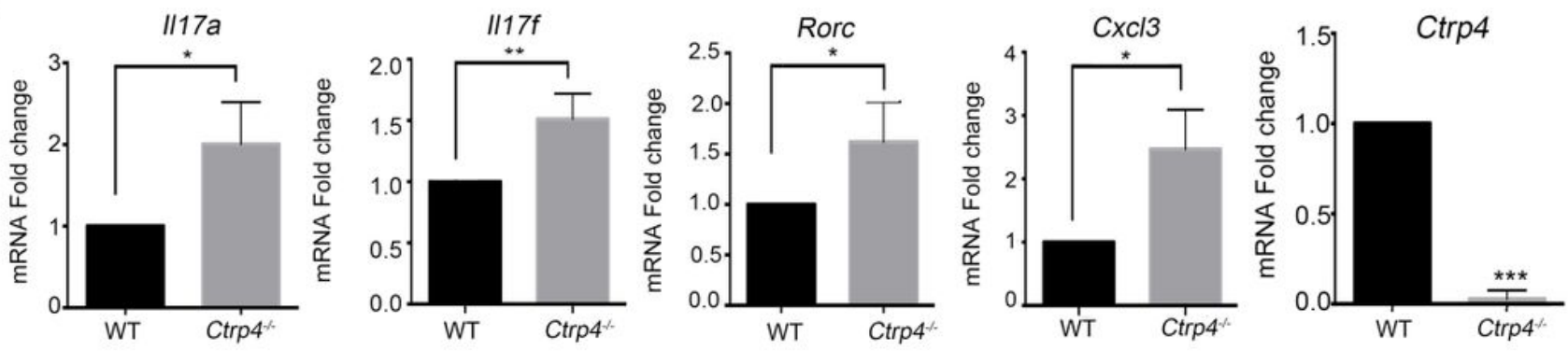

Figure 1

Ctrp4 deficiency perturbs T cell homeostasis (A)Surface staining of CD4 and CD8 on Ctrp4-/- or WT thymocytes. Numbers in quadrants indicate the percentage of different stage cells including double negative, double positive, CD8-CD4+ single positive and CD4-CD8+ single positive T cells ( $n=10$ animals per group from one representative experiment of three).(B)The transition between the different population of CD4-CD8- in the thymus is marked by surface staining of CD44,CD25,c-kit and CD24( $n=10$ animals per group from one representative experiment of three).(C)Representative plots showed the percentage of 
naïve(CD44lowCD62Lhi) and memory/effector (CD44hiCD62Llow)CD4+T cells in the spleen. (D)Flow cytometry analyses of the frequency of naïve(CD44lowCD62Lhi) and memory/effector (CD44hiCD62Llow) CD8+T cells in the spleen.(E) Flow cytometry analyses of Th1(IFNy+) and Th17(IL17A+)effector T cells in the spleen of Ctrp4-/- and WT mice ( $n=8 /$ group). Data are frequency of CD4+CD44+ cells and presented as representative plots and summary graphs of three independent experiments.(F)Gene expression of II17a,II17f,Rorc,Cxcl3,Ctrp4 mRNA in CD4+ T cells from Ctrp4-/- or WT mice ( $n=5 /$ group)were analyzed by quantitative real-time PCR. Values were normalized against Gapdh. Data are shown as mean \pm SEM and are from one of three independent experiments with similar results. Statistical significance was determined using two-tailed unpaired Student t-test, ${ }^{\star} P<0.05, \star \star P<0.001$,

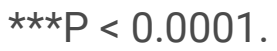

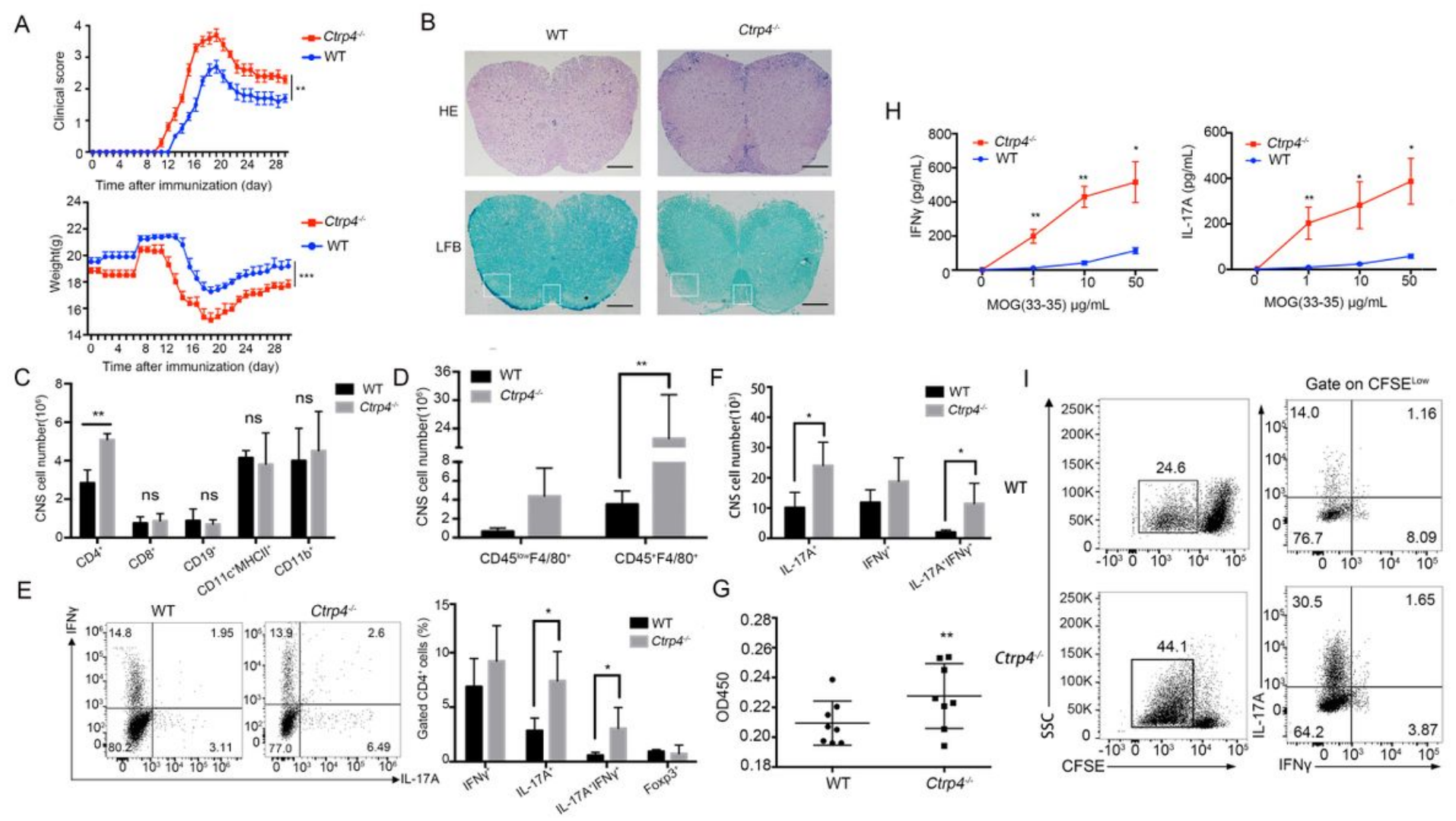

\section{Figure 2}

Ctrp4 deficiency increased EAE severity with CD4+T cells infiltration in the CNS. (A) After immunized with MOG peptide, the progression of disease was monitored and clinical EAE scores of Ctrp4- $-(n=10)$ or WT $(n=10)$ mice following disease induction were shown. ${ }^{*} \mathrm{P}<0.05, * * \mathrm{P}<0.001,{ }^{*} * \mathrm{P}<0.0001$ (two-way ANOVA with Bonferroni's post-test). (B)Spinal cord from Ctrp4-/- or WT mice were isolated 16 days after MOG immunization, and fixed and froze sections were obtained. Tissue section were stained with hematoxylin and eosin(H\&E) or luxol fast blue(LFB).Images are representative of three mice in each group.(C)Singe cells were isolated from the central nervous system on day 18 after EAE induction and stained with the indicated cell type-specific markers. Data represent mean absolute number. (D) Quantification of macrophage(CD45hiF4/80+) and microglia(CD45lowF4/80+) in CNS.(E)Representative contour plots showing the percentage of IFNY+ and IL-17A+ in CNS.(F)Absolute cell numbers of 
IFNy+CD4+, IL-17A+ CD4+, IFNy+ IL-17A+ CD4+ in CNS.(G)Enhanced T-cell response to MOG peptide were determined $72 \mathrm{~h}$ later. $(\mathrm{H})$ The production of IL-17A and IFN- $\gamma$ due to MOG stimulation were quantitated. (I)Representative flow plots of CD4+ T cells CFSE dilution after 5 days of in vitro restimulation with indicated MOG peptide. The percentage of IL-17+ and IFN- $\gamma+$ cells were gated on CSFElowCD4+T cells. Data are shown as mean \pm SEM and are from one of three independent experiments with similar results. (C-H)Statistical significance was determined using two-tailed unpaired Student $\mathrm{t}$-test, ${ }^{\star} \mathrm{P}<0.05$, ${ }^{\star *} \mathrm{P}<$ $0.001, * \star * P<0.0001$.
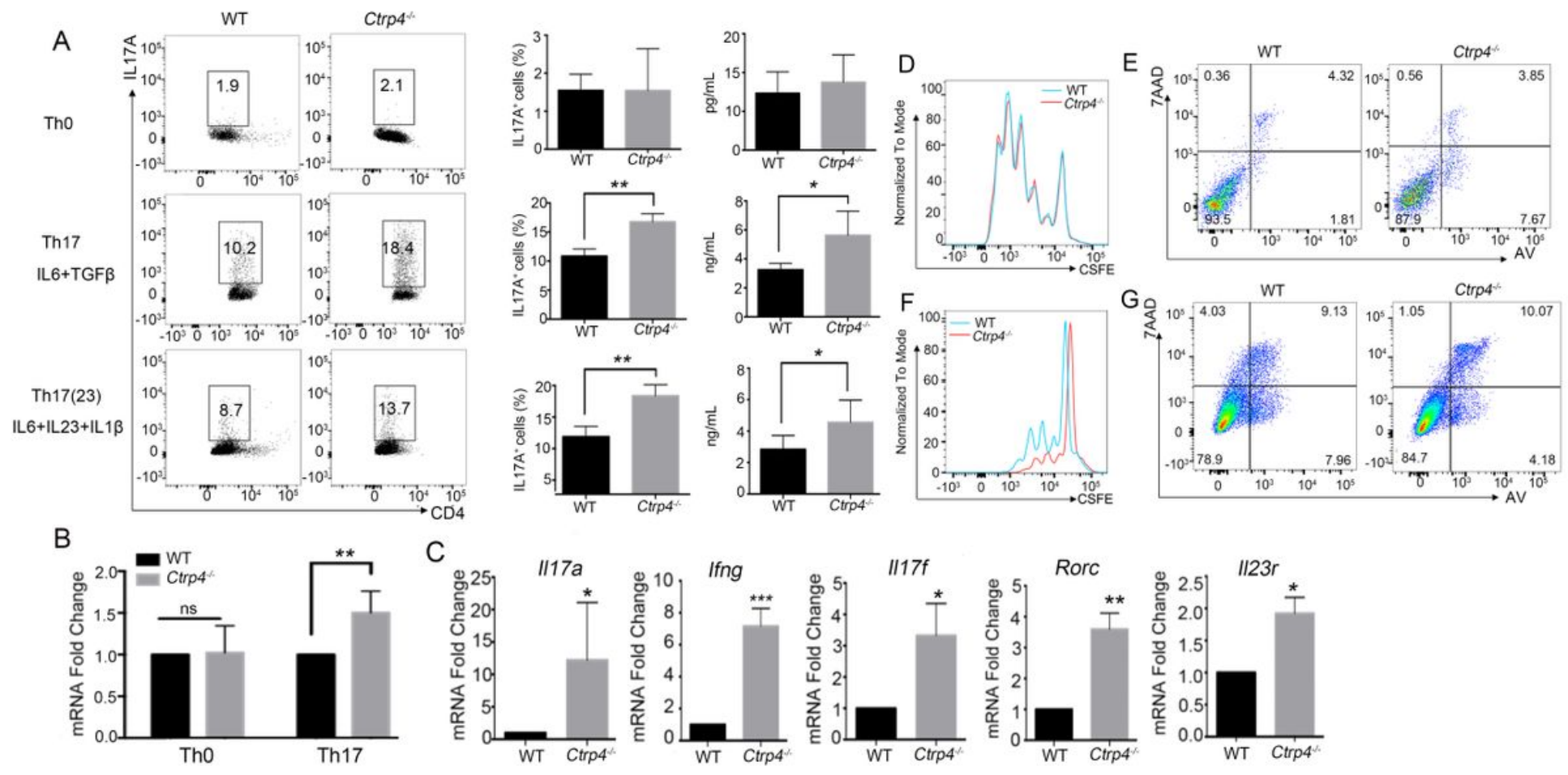

\section{Figure 3}

Th17 cell differentiation is impaired in Ctrp4-/- T mice A. Naive CD4+CD62LhighCD44lowCD25- T cells were sorted from WT and Ctrp4-/- miceA ( $n=5 /$ group), differentiated with (1)no cytokine,(2) TGF- $\beta 1$ and IL-6, or (3) IL-1 $\beta$, IL-6, and IL-23 and analyzed by intracellular cytokine staining after 4 days. One of five experiments is shown. The level of IL-17A in the supernatant were measured by ELASA (right).(B)The quantities of Rorc transcript were determined in WT and Ctrp4-/- T cells under Th0 or Th17 differentiation conditions(n=5/group).(C) Gene expression of II17a,II17f,Rorc,Ifng,II23r mRNA in CD4+ T cells under pathogenic Th17 differentiation condition from Ctrp4-/- or WT mice ( $n=5 /$ group) were analyzed by quantitative real-time PCR. Values were normalized against Gapdh. (D-E)Naïve T cells were stimulated for $72 \mathrm{~h}$ in the presence of TCR stimulations and assessed for proliferation(D) or apoptosis(E).(F-G)Flow cytometric analysis of CFSE dilution in restimulated $72 \mathrm{~h}$ Th17 cell cultures to detect proliferation(F) and apoptosis(G). Data are shown as mean \pm SEM and are from one of three independent experiments with similar results. (A-C)Statistical significance was determined using two-tailed unpaired Student t-test, *P < $0.05,{ }^{\star} * \mathrm{P}<0.001,{ }^{\star \star \star} \mathrm{P}<0.0001$. 

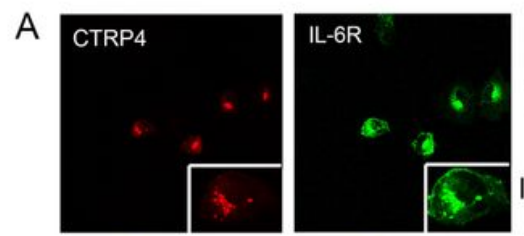

B

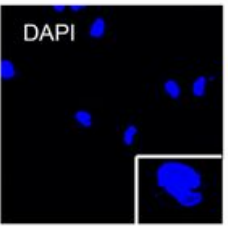

Merge

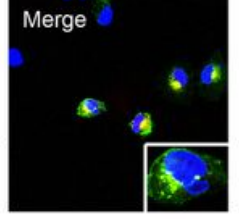

C

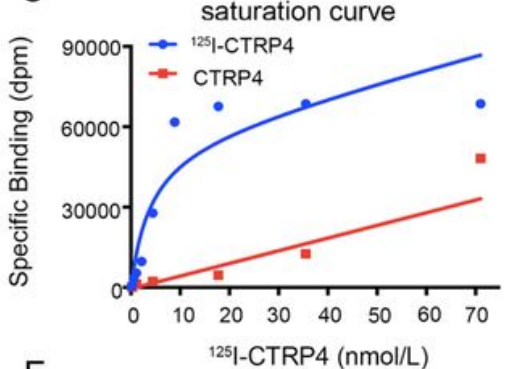

$\mathrm{F}$

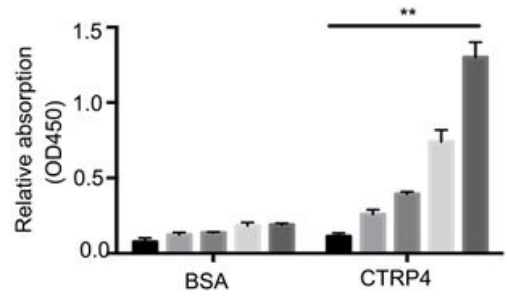

IB:IL-6R(55kDa)

IB:CTRP4(35kDa)
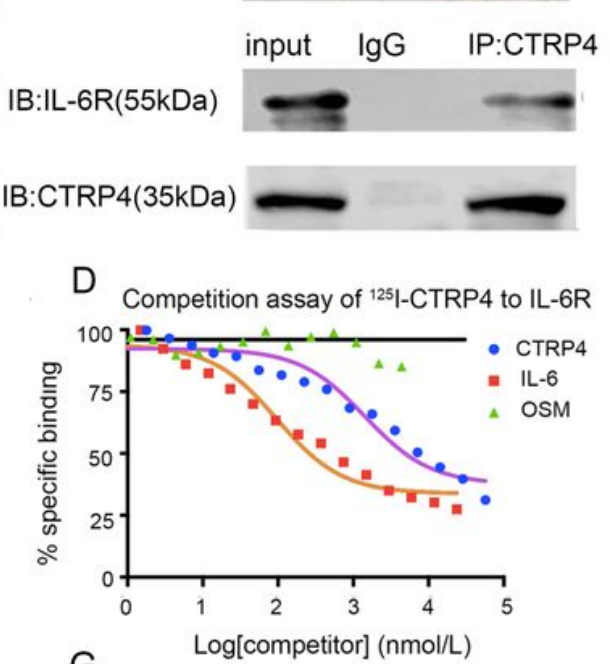

G

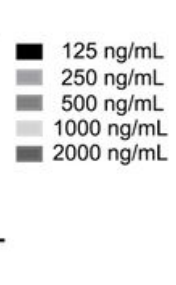

input IgG IP:IL-6R
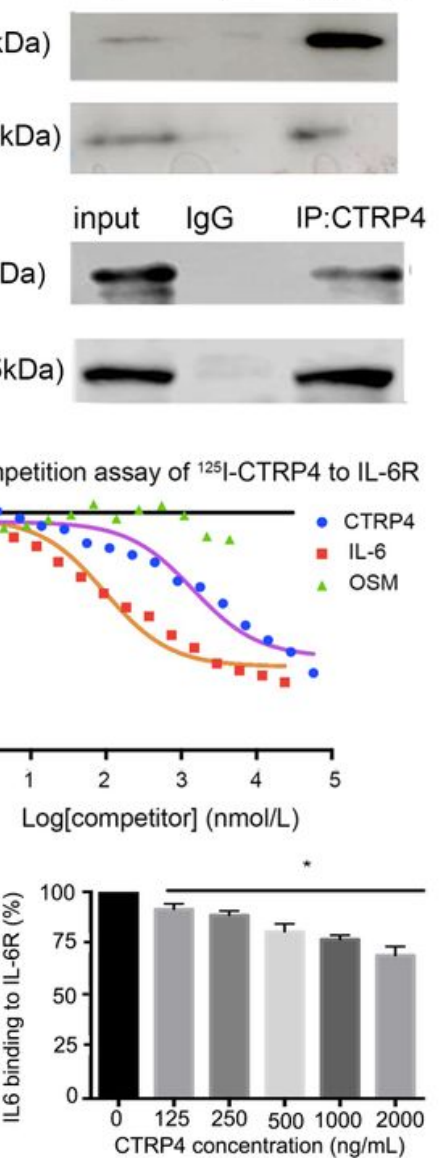

E

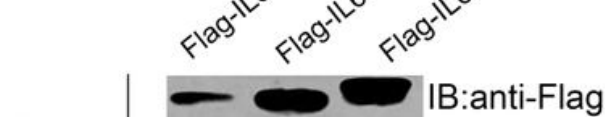

IP:Flag

IB:anti-myc

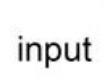

$=20$ IB:anti-Flag IB:anti-myc
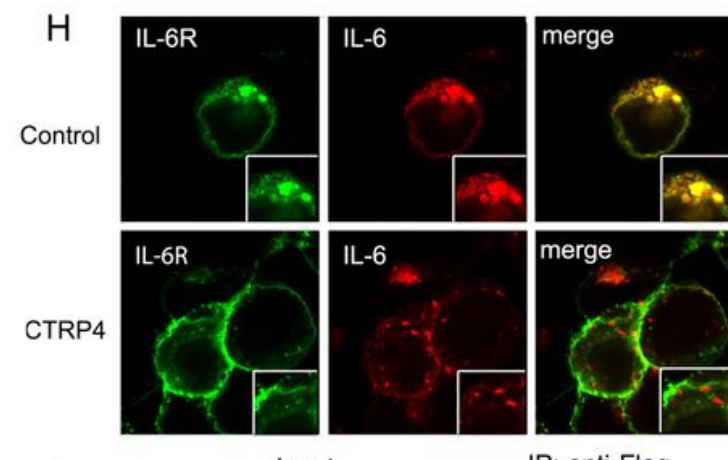

I

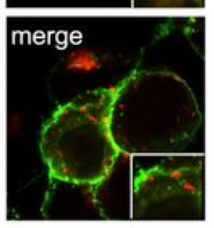

IP: anti-Flag

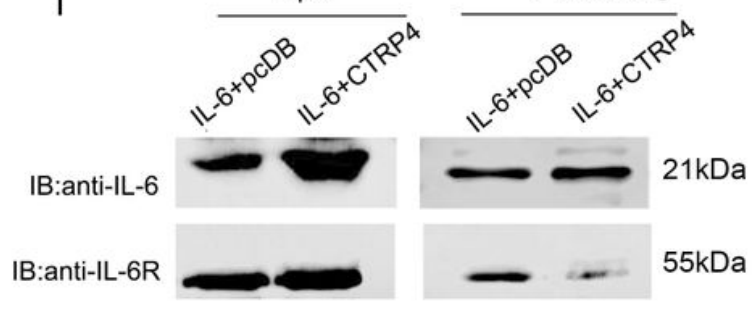

\section{Figure 4}

CTRP4 negatively regulates IL-6-induced STAT3 activation through IL-6-IL-6R axis (A) Jurkat cells were co-transfected with pmCherry-CTRP4 and EGFP-IL-6R and observed by laser confocal microscopy. Higher magnification images of boxed areas on lower power images are provided to the right of merged images.

(B) Jukat cell membrane lysates were immunoprecipitated with anti-IL-6R followed by analysis of indicated proteins (upper panel) or with anti-CTRP4 followed by analysis of indicated proteins (lower panel).(C)Saturation curves of CTRP4 binding to IL-6R. The curve respresents experiments performed in duplicate samples.(D) Competitive binding assay. The interaction between 125I-CTRP4 and IL-6R were disturbed by addition of unlabeled-CTRP4, IL- 6 and OSM as control.(E)HEK293T were transiently cotransfected different Flag-tagged IL-6R deletion mutants. Lysates were immunoprecipitated with antiFlag, followed by western analysis with anti-Flag and anti-Myc. (F)Increasing concentrations of rhCTRP4 were added to plate-bound IL-6R. Dose-dependent binding of CTRP4 to IL-6R was detected by anti-IL-6R mAb but was not observed in addition of BSA to IL-6R-coated plates. (G)Specificity of binding of soluble rhCTRP4 to solid-phase IL-6R was performed by competitive ELASA. Increasing concentrations of rhCTRP4 were incubated with a constant concentration $(300 \mathrm{ng} / \mathrm{mL})$ of IL- 6 and the mixture were added to solid-phase IL-6R plates. The binding was detected by anti-IL-6R mAb. Binding is expressed as the mean OD450 \pm S.D. of duplicate samples and is shown for one example representative of three 
independent experiments. (H-I) HEK293T cells were co-transfected with EGFP-IL-6R and pmCherry-IL-6 in the presence or absence of CTRP4 overexpression. The interaction of IL-6 to IL-6R were detected by laser confocal microscopy $(\mathrm{H})$ and co-immunoprecipitation $(I)$. $(F, G)$ Data are shown as mean \pm SEM and are from one of three independent experiments with similar results. Statistical significance was determined using two-way ANOVA with Bonferroni's post-test. ${ }^{*} \mathrm{p}<0.05 ;{ }^{* *} \mathrm{p}<0.01$.
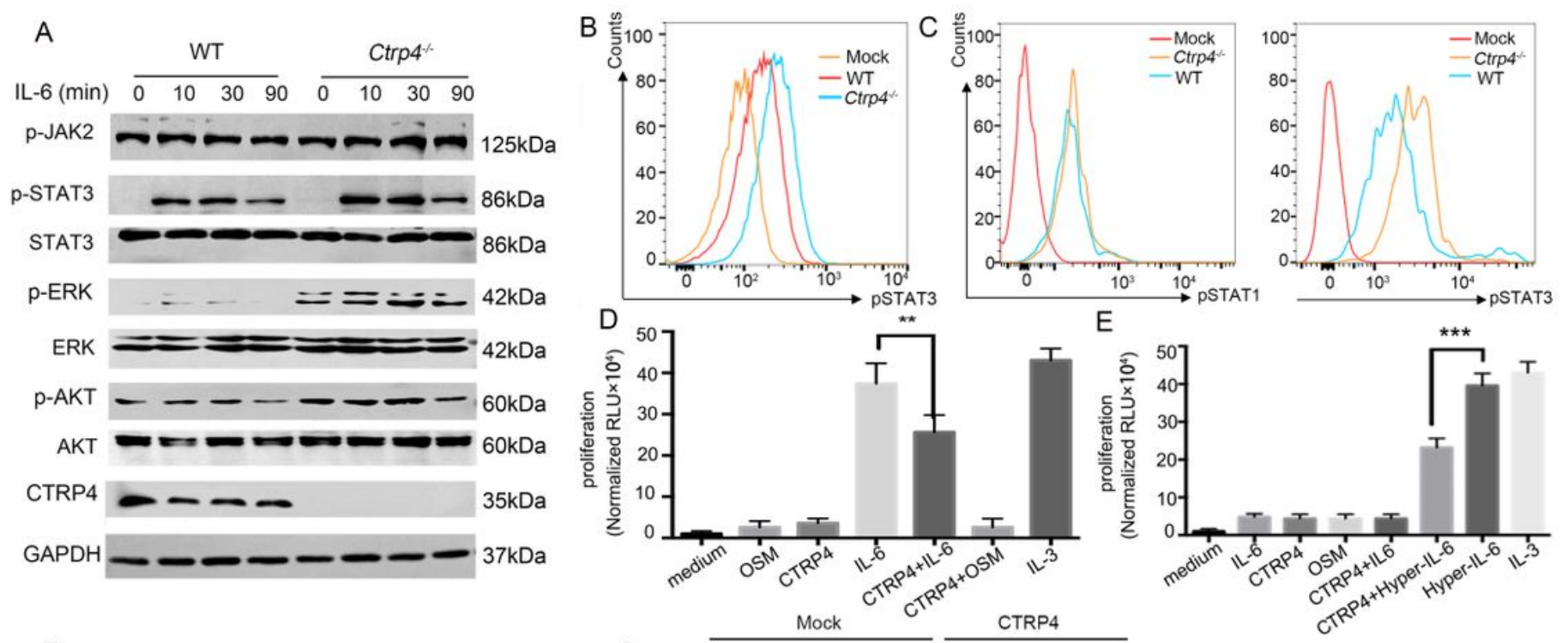

$\mathrm{F}$
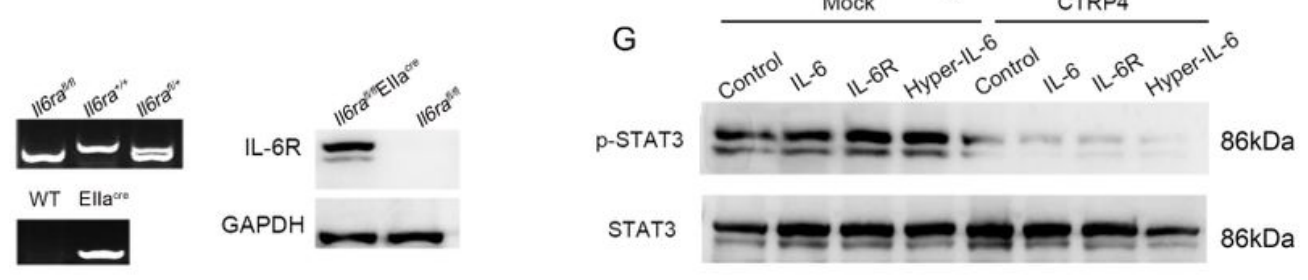

\section{Figure 5}

Mechanism underlying the inhibition of Th17 cell differentiation by CTRP4 (A)Purified CD4+T cells from Ctrp4-/- and WT mice were treated with IL-6 $(100 \mathrm{ng} / \mathrm{mL})$ for specific time. Lysates were probed for phosphorylated JAK2(p-JAK2), p-STAT3,STAT3,p-ERK,ERK,p-AKT,AKT,CTRP4 and GAPDH. (B) Freshly isolated WT and Ctrp4-/-CD4+T cells were treated with IL-6 $(100 \mathrm{ng} / \mathrm{mL})$ for $30 \mathrm{~min}$. The level of phosphor-STAT3 and total STAT3 were examined by flow cytometry.(C) Representative flow cytometry analysis of p-STAT3 or p-STAT1 from Ctrp4-/- and WT CD4+T cells at the peak of disease.(D) Proliferative response of Ba/F3-gp130 cells cultured for 2 days in the presence of exogenous $\mathrm{IL}-6(10 \mathrm{ng} / \mathrm{mL})$, OSM(100ng/mL),CTRP4(100ng/mL), CTRP4 plus IL-6, OSM plus IL-6 or IL-3(10ng/mL) or left untreated. Live cells were evaluated with the colorimetric CCK8 assay. (E) Proliferative response of Ba/F3-gp130-II6R cells cultured for 2 days with IL-6 $(10 \mathrm{ng} / \mathrm{mL})$, OSM $(100 \mathrm{ng} / \mathrm{mL})$, CTRP4 $(100 \mathrm{ng} / \mathrm{mL})$, hyper-IL-6 (50ng/mL), CTRP4 plus IL-6, CTRP4 plus hyper-IL-6 or IL-3 or left untreated. Data are mean values with SD of triplicate culture wells. Data are from one experiment representative of three independent experiments with similar results. (F) Genotyping by PCR analysis of $116 \mathrm{rafl} / \mathrm{fl}, \mathrm{il} 6 \mathrm{ra}+/+$ and heterozygous mice or PCR analysis of Ellacre mice.(G) Western blot analysis of p-STAT3 and STAT3 protein levels in \|6rafl/flEllacre MEF cells. Prior to analysis, Il6rafl/flEllacre MEF cells were stimulated with IL- 
$6(10 \mathrm{ng} / \mathrm{mL}), \mathrm{IL}-6 \mathrm{R}(10 \mathrm{ng} / \mathrm{mL})$ and hyper-IL-6 $(10 \mathrm{ng} / \mathrm{mL})$ for $1 \mathrm{hr}$. Representative of five independent experiments with 5 mice per experiment. Data are shown as mean \pm SEM and are from one of three independent experiments with similar results. (D,E)Statistical significance was determined using two-way ANOVA with Bonferroni's post-test. * $p<0.05 ;{ }^{* *} p<0.01$.

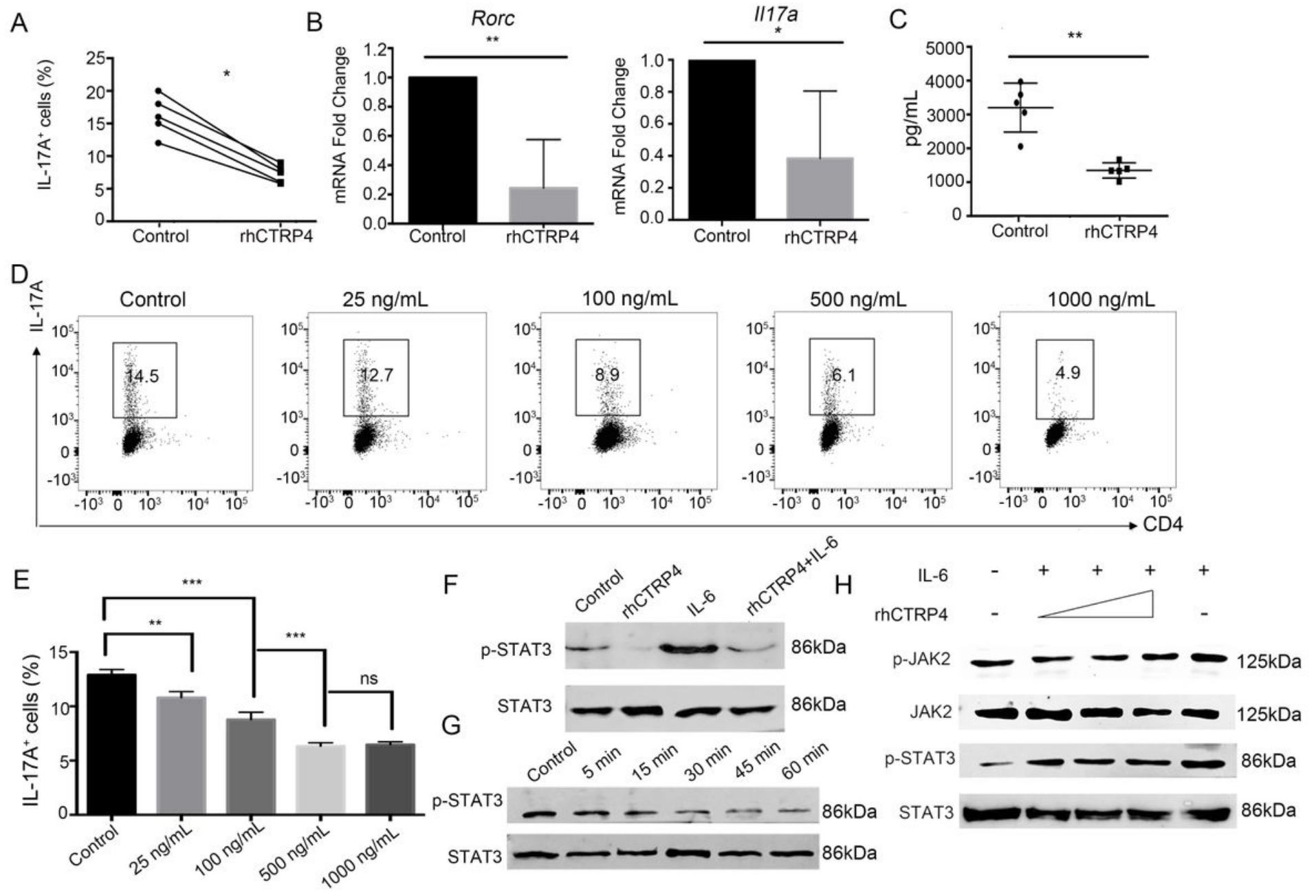

\section{Figure 6}

rhCTRP4 treatment inhibits IL-6-mediated STAT3 activation (A) Flow cytometry analysis of intracellular IL-17A from in vitro polarized WT CD4+T cells to Th17 in the presence of $100 \mathrm{ng} / \mathrm{mL}$ rhCTRP4 for 4 days followed by restimulation. Data are shown as mean \pm SEM. ${ }^{*}, \mathrm{p}<0.05$ versus WT group(paired Student $t$ test). $n=5$ mice per group and representative of three independent experiments (B) Gene expression of $\| 17 a$,Rorc mRNA in CD4+ T cells described as $(A)$ were analyzed by quantitative real-time PCR. Values were normalized against Gapdh. (C)The concentration of IL-17A in supernatant of cell cultures were analyzed by ELASA. (D-E) Purified WT naïve CD4+T cells were polarized under Th17 conditions with various dose of rhCTRP4 and cytokine production was analyzed $96 \mathrm{hr}$ later using flow cytometry. $(\mathrm{F})$ Purified WT CD 4+ cells were activated with IL-6 or with IL-6 prior to treatment with $100 \mathrm{ng} / \mathrm{mL} \mathrm{rhCTRP4}$ for $1 \mathrm{hr}$. Western blot was performed to analyze the activation of STAT3.(G)Time-dependent changes in the level of p-STAT3 as evaluated by western blot. (H) Purified WT CD4+ cells were activated with IL-6 or with IL-6 pretreated with various concentration of rhCTRP4(from $100 \mathrm{ng} / \mathrm{mL}$ to $1000 \mathrm{ng} / \mathrm{mL}$ ), followed by 
western blot. Representative of five independent experiments with 5 mice per experiment. Data are shown as mean \pm SEM and are from one of three independent experiments with similar results. (B,C) Statistical significance was determined using unpaired Student $t$ test; (E)Statistical significance was determined using two-way ANOVA with Bonferroni's post-test. ${ }^{*} p<0.05$; ${ }^{*} \mathrm{p}<0.01,{ }^{* \star *} \mathrm{p}<0.001$, ns not significance.
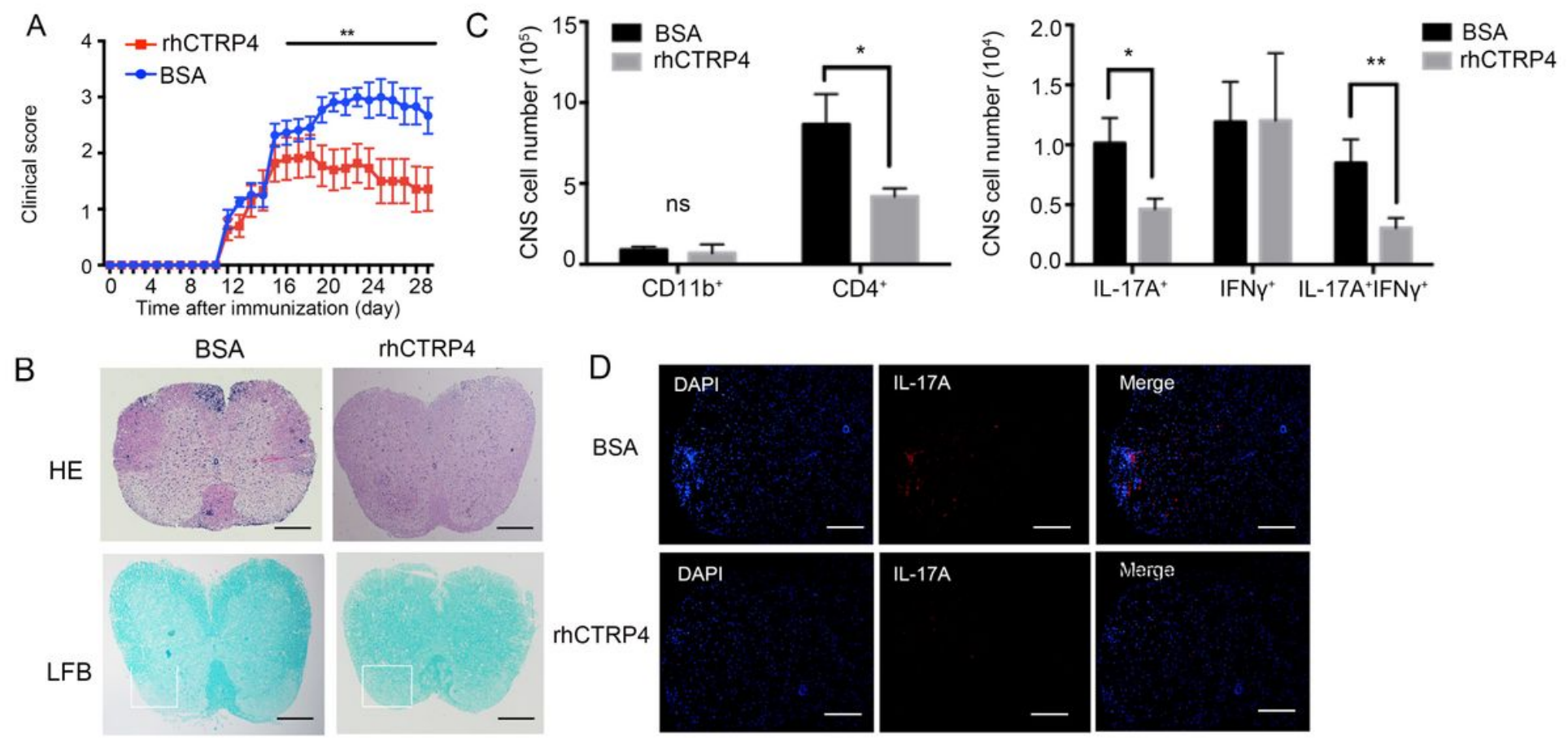

rhCTRP4
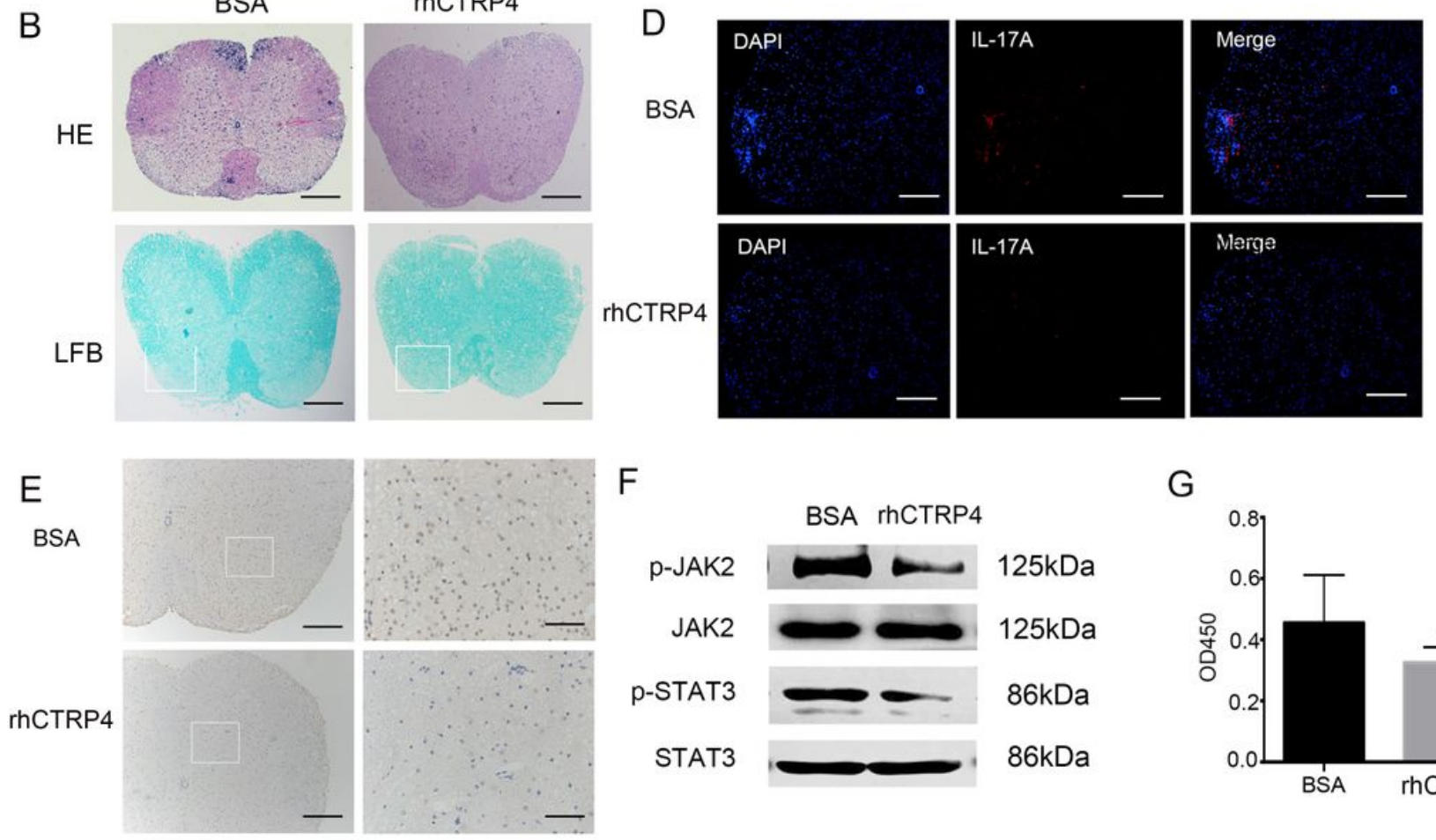

$\mathrm{F}$

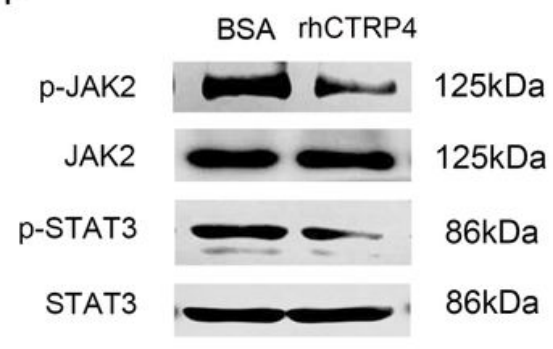

G

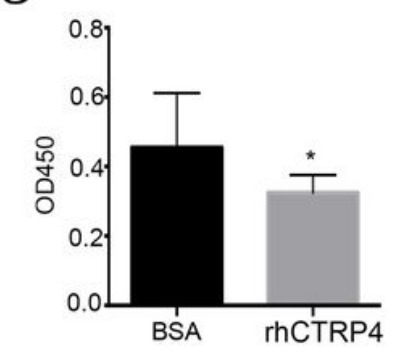

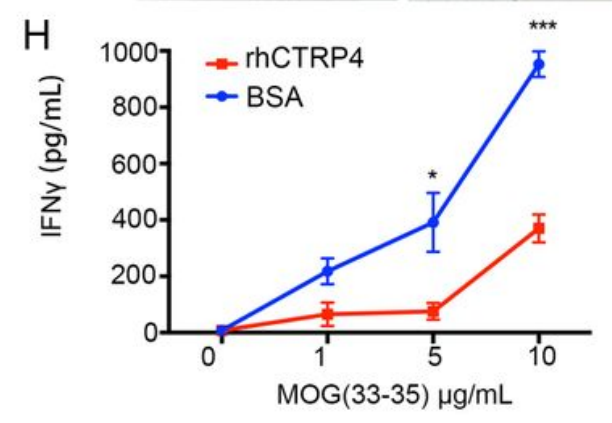

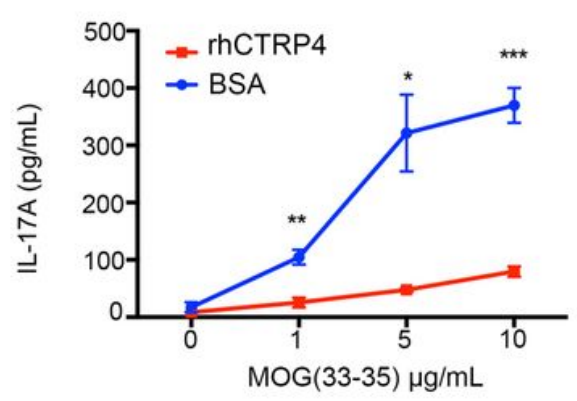

\section{Figure 7}

In vivo administration of rhCTRP4 attenuates the clinical severity (A)Active EAE was induced in C57BL/6 mice by subcutaneous immunization with MOG35-55 peptide in CFA and PTX injection.rhCTRP4 and control(BSA) were administered intraperitoneally on day onset (days 10) and every day until sacrificed. Clinical scores are depicted. $n=10 /$ group, ${ }^{* *} P<0.001$, Data are shown as mean $\pm S E M$ and analysed by 
two-way ANOVA with Bonferroni's post-test. (B)Representative hematoxylin/eosin(H\&E) and Luxol Fast Blue(LFB) staining on spinal cord sections. Scale bar: $200 \mu \mathrm{m}$, and $4 \times$ magnification. (C) Quantification of CD11b+ and CD4+ cells (left) and the number of IFNY+ and IL-17A+ in CNS(right). Data are shown as mean \pm SEM and analysed by unpaired student $t$ test (D) Immunofluorescent staining of IL-17A+ cells in the spinal cord. Nuclei were counterstain with DAPI. Scale bar: $100 \mu \mathrm{m}$, and 10x magnification.

(E)Immunohistochemistry detection of nuclear p-STAT3(Y705) within spinal cord of animals with EAE treated with BSA versus rhCTRP4. Scale bar: $100 \mu \mathrm{m}$, and 10x magnification. (F)Activity phosphorylation of STAT3 was detected in CD4+Tcells from Ctrp4-/- and WT mice 18 days after EAE induction. Pooled data are presented.(G)MOG35-55-specific proliferation of splenocytes that were isolated from rhCTRP4treated group or control group and re-challenged with MOG35-55 peptide was detected by BrdU.(F) The production of IL-17A and IFN- $\gamma$ due to MOG stimulation were quantitated. Data are shown as mean \pm SEM and are from one of three independent experiments with similar results. $(\mathrm{G}, \mathrm{H})$ Statistical significance was determined using unpaired Student $t$ test; ${ }^{*} p<0.05 ;{ }^{* *} p<0.01$, ${ }^{* \star} p<0.001$, ns not significance.

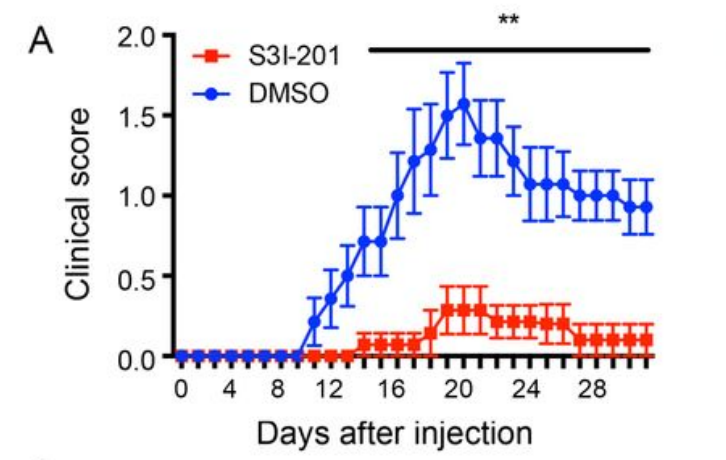

B

DMSO

S3I-201
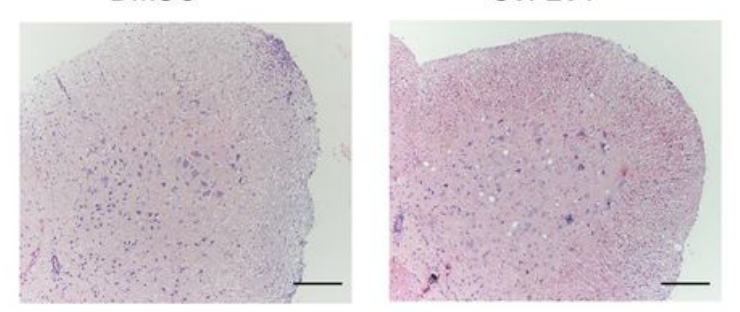

C

BSA
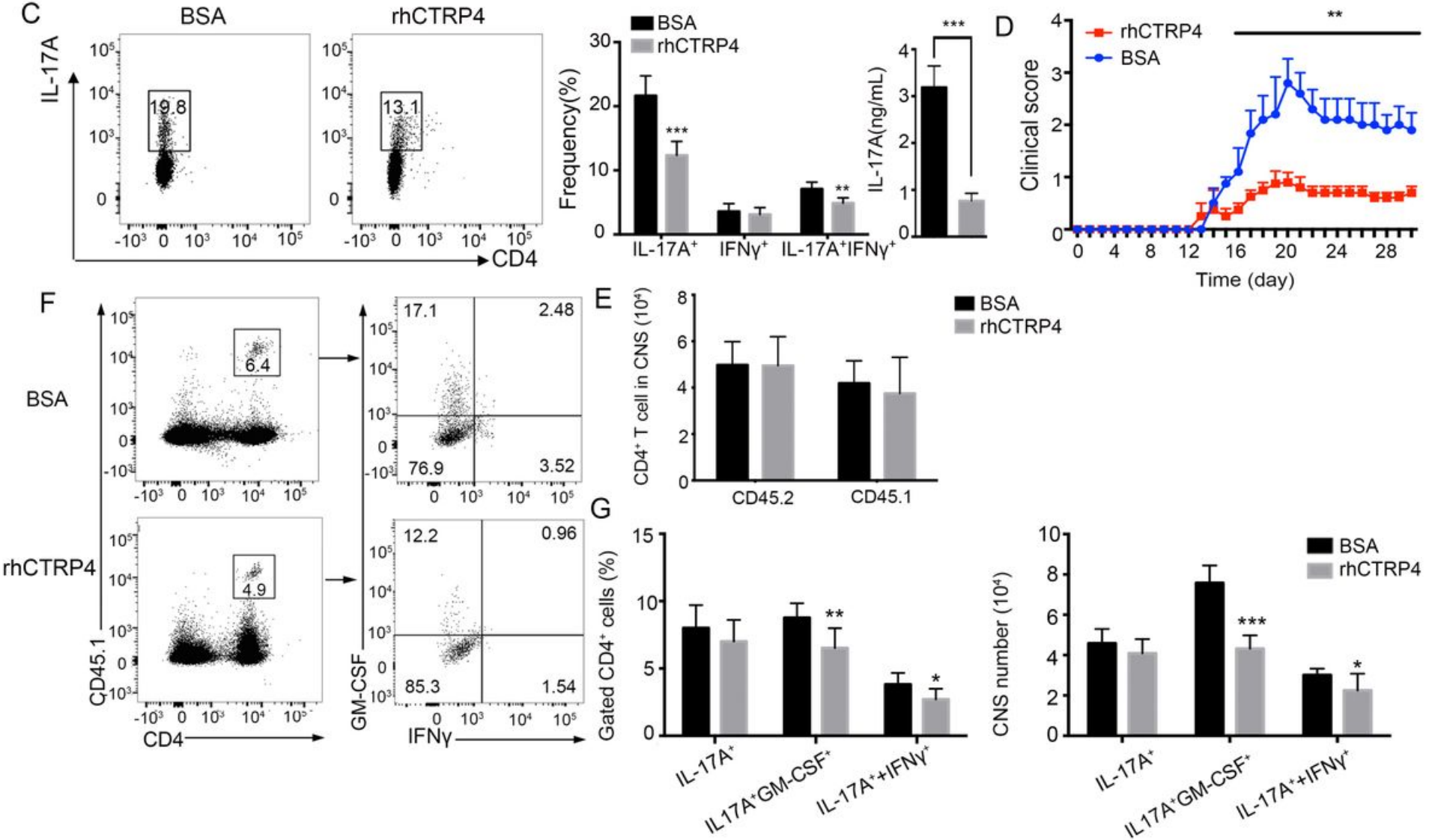

BSA

rhCTRP4
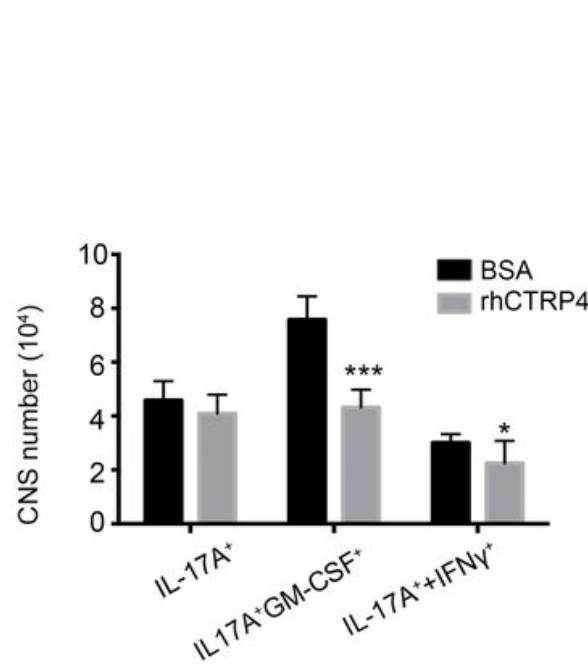


\section{Figure 8}

rhCTRP4 affects the pathogenicity of MOG-reactive CD4+T cells (A) Ctrp4-/- mice subjected to MOGinduced EAE were treated with DMSO or S3I-201.Each group was monitored and scored daily. $n=10 /$ group, $* * P<0.001$, Data are shown as mean \pm SEM and analysed by two-way ANOVA with Bonferroni's post-test. (B)Histopathology of spinal cord from two group by H\&E staining. Scale bar: 100 $\mu \mathrm{m}$, and 10x magnification. (C)Lymphoid cells from the draining LNs of B6.SJL mice(CD45.1+) that were previously immunized with MOG35-55 in CFA were rechallenged with the same peptide in the presence of BSA or rhCTRP4 and IL-23. Representative FACS plots and graphs summarizing the frequency of CD4+IL$17 A+, C D 4+I F N Y+$ and $C D 4+I L-17 A+I F N Y+(L e f t$ panel). The level of IL-17A in the supernatant were measured by ELASA (right).(D-G)The ex-vivo-expanded MOG-specific CD4+ pretreated with BSA or rhCTRP4 were i.v. adoptively transferred into irradiated congenic recipients(CD45.2).(D) The clinical scores of EAE development. $n=12 /$ group, ${ }^{*} P<0.001$, Data are shown as mean $\pm S E M$ and analysed by two-way ANOVA with Bonferroni's post-test. (E)The absolute number of CD4+T cells in CNS.(F and $G)$ The frequencies and absolute numbers of CD4+IL-17+, CD4+IL-17+IFNY+, CD4+IL-17+GM-CSF+ donor cells in the CNS tissue. Data are shown as mean \pm SEM and are from one of three independent experiments with similar results. Statistical significance was determined using unpaired Student $t$ test; ${ }^{\star} p<0.05 ;{ }^{* \star} p<0.01$, $\star * \star p<0.001$, ns not significance.

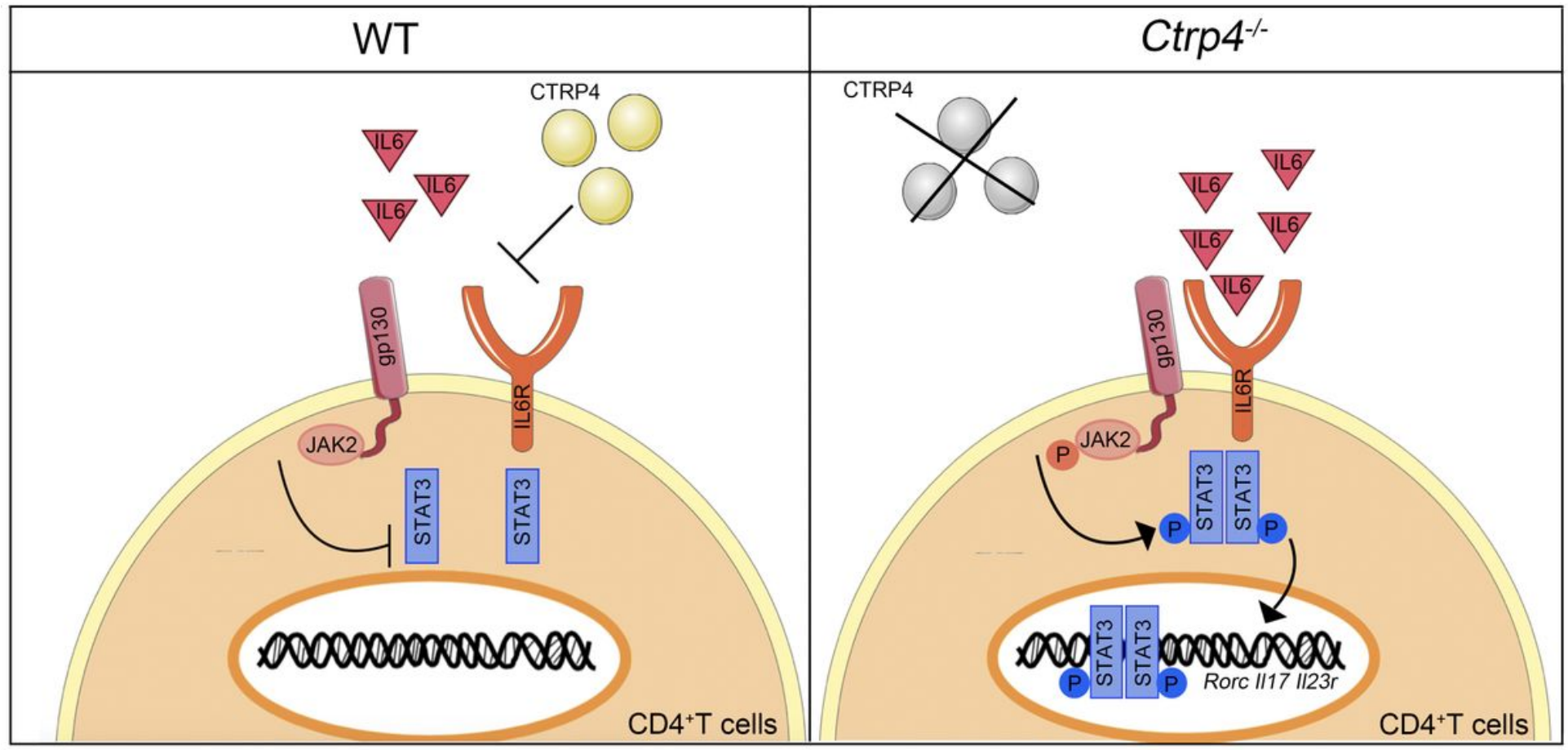

\section{Figure 9}

Schematic diagram represents the role of CTRP4 in naïve CD4+ T cells, in which CTRP4 inhibits the binding of IL- 6 and IL-6R by blocking downstream STAT3 pathway Th17 differentiation is dependent on IL- 6 inducing activation of STAT3 transcriptional factor. STAT3 induces the expression of the "master regulator" gene of Th17 cell differentiation, RORyt, which then induces the expression of Th17 effector 
cytokine such as IL-17A. Loss of CTRP4 promotes IL-6-mediated IL-6R-gp130 complex formation, recruitment and phosphorylation of JAK2, with subsequent phosphorylation and nuclear localization of STAT3 to activate STAT3 transcription targets, leading to much more severe clinical disease symptom in Ctrp4-/- mice.

\section{Supplementary Files}

This is a list of supplementary files associated with this preprint. Click to download.

- supplementaltable1.tif

- supplementaryfigure1.tif

- supplementalfigure2.tif

- supplementalfigure3.tif

- supplementalfigure4.tif

- supplementalfigure5.tif 